\title{
Museum collections indicate bird defaunation in a biodiversity hotspot
}

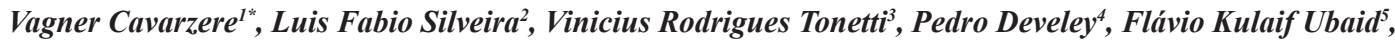 \\ Luciano Bonatti Regalado ${ }^{6}$ \& Luiz Fernando de Andrade Figueiredo ${ }^{7}$ \\ ${ }^{1}$ Universidade Tecnológica Federal do Paraná, Prolongamento da Rua Cerejeira, 85892-000, Santa Helena, PR, \\ Brazil \\ ${ }^{2}$ Universidade de São Paulo, Museu de Zoologia, Seção de Aves, Avenida Nazaré, 04263-000, São Paulo, SP, Brazil \\ ${ }^{3}$ Universidade Estadual Paulista, Departamento de Ecologia, Campus de Rio Claro, Avenida 24a, 13506-900, \\ Rio Claro, SP, Brazil \\ ${ }^{4}$ BirdLife/SAVE Brasil, São Paulo, SP, Brazil \\ ${ }^{5}$ Universidade Estadual do Maranhão, Departamento de Química e Biologia, Morro do Alecrim, Caxias, MA, Brazil \\ ${ }^{6}$ Instituto Chico Mendes de Conservação da Biodiversidade, Floresta Nacional de Ipanema, Caixa Postal 217, \\ Centro, CEP 18190-000, Araçoiaba da Serra, SP, Brazil \\ ${ }^{7}$ Centro de Estudos Ornitológicos, São Paulo, SP, Brazil \\ *Corresponding author: Vagner Cavarzere, e-mail: vagnera@utfpr.edu.br
}

CAVARZERE, V., SILVEIRA, L. F., TONETTI, V. R., DEVELEY, P., UBAID, F. K., REGALADO, L. B., FIGUEIREDO, L. F. A. Museum collections indicate bird defaunation in a biodiversity hotspot. Biota Neotropica. 17(4): e20170404. http://dx.doi.org/10.1590/1676-0611-BN-2017-0404

\begin{abstract}
Ipanema National Forest, southeastern Brazil, once contained 340 bird species. Forest cover suffered for centuries from log exploitation and, as a result, most of the remaining forests are now an impoverished subset of the original vegetation. We show how the bird community changed over time by comparing historical and recent records. Currently, 228 species can be recorded, for a compilation of 410 species, of which 359 are documented. Some 89 forest species with historical records failed to be detected in recent surveys. Of the 72 Atlantic Forest or Cerrado endemic species, no more than 29 (40\%) are still found. The bird community changed from one which used to be related to coastline rain forests to another, which relates more to drier semideciduous forests of the interior.
\end{abstract}

Keywords: Atlantic Forest, Cerrado, hierarchical cluster analysis, multivariate analysis, semideciduous forests.

\section{Coleções de museus indicam defaunação de aves em um hotspot de biodiversidade}

Resumo: A Floresta Nacional de Ipanema, sudeste do Brasil, já abrigou 340 espécies de aves. Sua cobertura florestal sofreu por séculos com a exploração de madeira e, desse modo, a maior parte da vegetação remanescente é uma sub-representação daquela original. Neste artigo é demonstrado como a comunidade de aves foi modificada com o passar do tempo por meio da comparação entre registros históricos e recentes. Atualmente, 228 espécies podem ser registradas, para um total de 410 espécies, das quais 359 possuem documentação. Das espécies registradas historicamente, 89 não foram mais detectadas. Das 72 espécies endêmicas da Mata Atlântica ou do Cerrado, apenas 29 (40\%) ainda podem ser encontradas. A comunidade de aves, outrora similar à de florestas ombrófilas costeiras, atualmente é mais relaciona à comunidade de matas semideciduais mais secas do interior.

Palavras-chave: Análise hierárquica de cluster, Análise multivariada, Cerrado, Floresta Semidecidual, Mata Atlântica.

\section{Introduction}

Defaunation, i.e., the process of losing animal species, has been accelerated in the last five centuries, a fact by which some authors have contended that earth is experiencing a "sixth extinction wave" (Barnosky et al. 2011, Pimm et al. 2014, Ceballos et al. 2015, 2017). Besides extinctions, abundance of vertebrates is sharply declining, leading to functional extinction of several species (Butchart et al. 2010, Ceballos et al. 2017). Among different causes of this phenomenon (e.g. hunting, pollution, impacts from invasive species and climate change), habitat loss is one of the main drivers, especially in tropical regions, where deforestation rates are high (Dirzo et al. 2014, Johnson et al 2017). Among the different vertebrate groups suffering species loss and population decline, birds, which suffer population decline worldwide (especially accentuated in tropical regions, Ceballos et al. 2017), play important roles in ecosystems, such as pollination, pest control and seed dispersal (Whelan et al. 2008). In addition to the loss of important 
services, the local extinction and population decline of birds can cause changes in evolutionary processes (Galetti et al. 2013).

Although one can infer bird species loss of a given degraded area by comparing its community to nearby areas, where species composition is assumed original, few studies have documented the disappearance of species over time at a same locality (but see Blake \& Loiselle 2015, 2016). Curtis \& Robinson (2015) resurveyed sites in Oregon from a 60-year-old historic dataset, comparing bird communities of the same sites between this time interval. In Neotropical regions, due to rare long-term data or earlier surveys, researchers have tried to assess extinction rates by comparing the composition of pre- and post-fragmentation bird assemblages (Willis 1974, 1979, Leck 1979, Bierregaard \& Lovejoy 1989). Few authors compared censuses separated by long time intervals. These are from little-surveyed sites at time lags of: 200 years in eastern Amazonia (Moura et al. 2014), 167 years in southwestern Amazonia (Silveira \& D'horta, 2002), 130 years in eastern Brazil (Christiansen \& Pitter 1997) and 100 years in Singapore (Sodhi et al. 2005). There are also examples of less than a century separating surveys, such as Barro Colorado Island, Panama (85 years, Robinson 1999), San Antonio, Colombia (80 years, Kattan et al. 1994), and the Viçosa region, Brazil (70 years, Ribon et al. 2003).

At Ipanema National Forest (hereafter INF), interior São Paulo state, almost 200 years of continuous and severe deforestation led to conspicuous habitat change. Many naturalists visited this iron foundry, including Johann Baptist von Spix and Karl Friedrich Philip von Martius (Spix \& Martius 1824), Friedrich Sellow and Ignaz Franz Werner Maria von Olfers (Stresemann 1948), Baron Georg Heinrich von Langsdorff, Peter Wilhelm Lund and Johannes Reinhardt and Johann Christian Mikan (Mikan 1820, Reinhardt 1870, Pinto 1979, Krabbe 2007). No other naturalist, however, contributed more to the knowledge of this locality than Johann Natterer, whose zoological work has no parallel in the history of scientific expeditions in Brazil (Pinto 1979). Thus, the site's well-documented (as museum specimens collected by naturalists) original avifauna provides a singular opportunity to evaluate how habitat loss has changed this Atlantic Forest bird community over time.

We compiled all bird records from the INF. We searched for both historical (museum specimens acquired during the nineteenth century naturalists' expeditions) and recent records, which included field data we have been gathering since 1986 and publications. We specifically addressed the question whether recent bird species composition in a highly surveyed biodiversity hotspot differed from that of 200 years ago by comparing species recorded in the 1800 s to species recorded in recent surveys. We used similarity indices and cluster analysis comparing species richness at the INF to other well-surveyed rain and semideciduous forests wishing to determine to which of these forests the INF used to be more closely related. We then conducted these same analyses only with the species we recently recorded at the INF to determine whether species richness changed over time. We hypothesized species richness changed considerably from one closely related to rain forests to another, which is more similar to semidecidual forests.

\section{Material and Methods}

\section{Study area}

INF $\left(23^{\circ} 26^{\prime}\right.$ S, $\left.47^{\circ} 36^{\prime} \mathrm{W}\right)$ is located in the state of São Paulo, southeastern Brazil, within the municipalities of Araçoiaba da Serra, Capela do Alto and Iperó, ca $120 \mathrm{~km}$ west of the city of São Paulo (Figure 1). The region is predominantly inserted within the Atlantic Forest domain with some Cerrado enclaves. These two domains are biodiversity hotspots that, despite harboring several endemic and threatened species have suffered from intense habitat loss (Ribeiro et al. 2009, Strassburg et al. 2017). INF is drained by the Sapucaí River to the west and Ipanema River to the east, with elevations ranging from 550-970 $\mathrm{m}$ asl. According to Spix \& Martius (1824:52), during the 1800 s the vegetation cover was "almost everywhere covered with thick woods (...) The forests, which stand more luxuriant and thicker in the hollow than in the higher parts, are uncommonly rich in the most various kinds of wood". Currently, it encompasses $\sim 5,000$ ha, of which $75 \%$ are covered by semideciduous ( $90 \%$ of all forests) and rain forests in several successional stages $(\sim 2,800$ ha), secondary growth and savannas, including ecotones between semideciduous forests and Cerrado sensu lato ( $\sim 540$ ha), water reservoirs and dams $(\sim 250$ ha) and native reforestations ( 220 ha, Radambrasil [1983]). The current vegetation is composed of secondary forests due to the extensive exploitation of tree species in the last two centuries to fuel the iron foundry furnaces (Saint-Hilaire 1976). Climate is considered as Cfa in Koeppen's classification, with defined wet (September-February) and dry (March-August) seasons. Annual mean rainfall is $1,400 \mathrm{~mm}$, whereas mean annual temperature is $20.4^{\circ} \mathrm{C}$, with a minimum of $11.6 \mathrm{C}^{\circ}$ in July and reaching $29.8 \mathrm{C}^{\circ}$ in February (MMA 2003). The land cover surrounding forests comprise water reservoirs and dams, regrowth, recovery of degraded areas, settlements of rural workers, the administrative headquarters and preserved historical sites, such as ruins of the foundry (MMA 2003).

The vegetation at INF suffered from tree exploitation since the beginning of the iron foundry (Saint-Hilaire 1976). Intensive deforestation began during the nineteenth century, when metal craft was transformed into a metallurgical industry at the cost of large-scale exploitation of mines at the Araçoiaba Mountain between 1811-1833 (Menon 1992). Due to Brazilian governmental orders, as much as 2,764 tons of charcoal fueled auto-ovens during the Brazil-Paraguay war in 1878 (Dupré 1885). Between 1926 and 1943, the Araçoiaba Mountain suffered from apatite extraction; during the 1950s the dominant activity was the production of cement, still produced today. It was only in May 1992, when the area was legally declared a National Forest (which still allows the selective use of natural resources according to Brazilian law), that deforestation stopped within the INF and $\sim 350$ hectares were destined for fast growing Eucalyptus plantations ( $200 \mathrm{ha})$ and reforestations with native species ( 150 ha, MMA 2003).

\section{Bird records}

\subsection{Historical survey}

At that time naturalists provided few details on bird collection data. Species collected by Natterer are museum specimens that are predominantly deposited at the Naturhistorisches Museum in Vienna, Austria. Refer to Appendix I.

\subsection{Recent survey}

We surveyed forest birds using point counts (Bibby et al. 2000) ever three months from May 2001-February 2002 and from August 2009-May 2013. We determined three unlimited-radius 10 -min point counts at least $200 \mathrm{~m}$ apart on two existing trails (north and south) inside mature forest within the INF, which we visited on four consecutive days following the same sequence of points. Therefore, each campaign consisted of 12 point counts (if inclement weather did not impede us from surveying). Although there are representative portions of the forest block outside the limit of the INF, all point counts lay within this protected area. We also used eight mist nets (12 m x $3 \mathrm{~m} \mathrm{x} 32 \mathrm{~mm}$ ) inside forests to record secretive species during 3 consecutive days on the 2001 and 2002 campaigns. Nets touched bottom and remained open from 06:00-17:00 h. We checked nets at 1-hr intervals and released captured birds after identification. We performed non-systematic surveys with transect counts (Bibby et al. 2000) on 
$47^{\circ} 40^{\prime} \mathrm{W}$

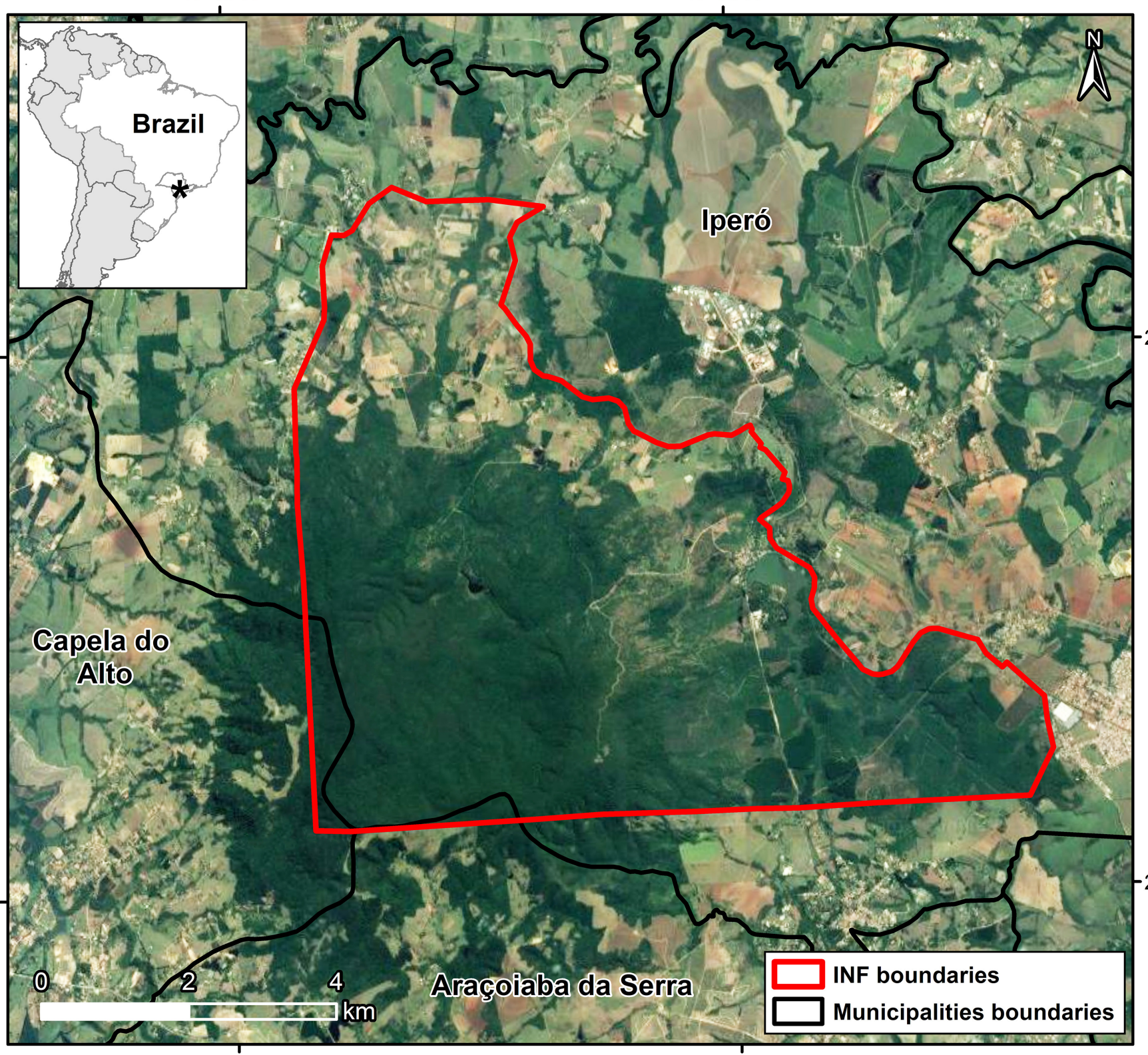

Figure 1. Location of the Ipanema National Forest (limited by a continuous line) within three municipalities in the state of São Paulo, southeastern Brazil.

26 July 2009, 14 November 2010, 21-22 July 2012 and 01 May 2013. To detect border and canopy species or flying-over birds (Robinson 1999) we further considered qualitative records conducted during ad libitum observations during the mornings and while moving between point counting. We walked randomly through different types of habitats, such as reed beds, marshes, pastures, capoeiras (early successional forests) and forest edges during mornings and afternoons (15:00-19:00 h). We surveyed different successional stages of vegetation, such as early succession, 5- to 10 -year-old regrowth and mature secondary forests. We detected birds $15 \mathrm{~min}$ before sunrise with the aid of binoculars and/or by vocalizations for both the point count method and random observations, accumulating 624 point counts $(104 \mathrm{~h})$, approximately $540 \mathrm{~h}$ of random observations and 600 mist-netting hours, more than any recent bird censuses (Table 1).

To complement our results, we searched for birds recorded within the INF in Web of Science (https://www.webofknowledge.com) and Google
Scholar (http://scholar.google.com.br) and included a few recent records from colleagues (Appendix II). In addition, we searched for "Floresta Nacional de Ipanema" archived digital vouchers on the internet on Wiki Aves (www.wikiaves.com.br) and on the global avian sound library Xeno-canto (www.xeno-canto.org). We did not consider misidentifications (records of species which do not conform to expected range) of published records. These included the record of counterparts from different biogeographic regions and the absence of the species which would occur in southern Brazil: Rusty-margined Flycatcher Myiozetetes cayanensis, Pied Water-Tyrant Fluvicola pica, and Cinereous Becard Pachyramphus rufus certainly correspond to the Social Flycatcher M. similis, Black-backed Water-Tyrant F. albiventer and the White-winged Becard P. polychopterus, respectively. Both the Blue-back Grosbeak Cyanoloxia cyanoides and the Red-breasted Blackbird Sturnella militaris were mistaken for their southern counterparts, the Ultramarine Grosbeak C. brissonii and the White-browed Blackbird 
Table 1. Source of historical bird records and detailed information on recent bird records at Ipanema National Forest, São Paulo, southeastern Brazil. For question marks, refer to Results. An asterisk indicates three misidentified species.

\begin{tabular}{|c|c|c|c|}
\hline Source & Date & $\begin{array}{c}\text { Time } \\
\text { spent }\end{array}$ & $\begin{array}{l}\text { Number } \\
\text { of species }\end{array}$ \\
\hline $\begin{array}{l}\text { J. B. Spix and } \\
\text { K. P. F. Martius }\end{array}$ & Jan 1818 & - & $13 ?$ \\
\hline J. C. Mikan & $1816-1817$ & - & $?$ \\
\hline $\begin{array}{l}\text { F. Sellow and } \\
\text { I. F. W. M. Olfers }\end{array}$ & Dec 1819 - Jan 1820 & - & $4 ?$ \\
\hline J. Natterer & $\begin{array}{l}\text { Feb } 1819 \text { - Jul } 1820 \text { / } \\
\text { Sep } 1821 \text { - Sep } 1822\end{array}$ & - & 340 \\
\hline J. H. Langsdorff & 1825 & - & $?$ \\
\hline $\begin{array}{l}\text { P. W. Lund and J. T. } \\
\text { Reinhardt }\end{array}$ & Mar 1834 & - & 7 \\
\hline C. Silva and L. B. Regalado & January 1995 & - & 1 \\
\hline L. B. Regalado & 1991-1996 & $128 \mathrm{~h}$ & 39 \\
\hline Y. Oniki \& E. O. Willis & 1986 & $2 \mathrm{~h}$ & 45 \\
\hline C. Silva & 1993-2003 & $350 \mathrm{~h}$ & $186^{*}$ \\
\hline Present authors & 1986-2015 & $1,244 \mathrm{~h}$ & 228 \\
\hline
\end{tabular}

S. superciliaris. For the most part, museum specimens identified by Pelzeln (1868) collected by nineteenth century naturalists accounted for our "historical records". Taxonomic arrangements follow the Brazilian Committee of Ornithological Records (Piacentini et al. 2015). Atlantic Forest endemic species are according to Parker et al. (1996) and Cerrado endemic species follow Silva \& Bates (2002).

\section{Analysis}

To investigate to which protected forests in São Paulo the INF were most similar regarding its historic bird species richness (presence/absence) we performed a hierarchical cluster analysis using Euclidean distance within the R environment (R Development Core Team 2015). We then ran this analysis with the current species richness of the INF. We chose Ward's minimum variance criterion as the objective function, defining which clusters merge at each step (Ward-Jr 1963). We also calculated Jaccard Dissimilarity Index (package vegan, function vegdist()), in which case lower values suggest higher similarities. We decided to compare INF with sufficiently well surveyed protected forests at least $250 \mathrm{~km}$ away. These included well surveyed (1) semideciduous forest with Cerrado enclaves in Lençóis Paulista (Donatelli et al. 2004), (2) semideciduous forests without Cerrado enclaves in Barreiro Rico Farm (Magalhães 1999) and Caetetus Ecological Station (Cavarzere et al. 2009) and (3) rain forests to the east of the INF - Boraceia Biological Station (Cavarzere et al. 2010), Serra do Mar State Park (Simpson et al. 2012) and to the west of the INF - Intervales State Park, (Vielliard \& Silva 2001) and Carlos Botelho State Park (Antunes et al. 2013; Table 2; Figure 2).

\section{Results}

\section{Recent records}

Compared to the 340 bird species INF once harbored, we recorded 228 species of 21 orders and 51 families. The compilation of all records from the INF resulted in a list of 410 species of 23 orders and 68 families, of which 359 are documented in the form of museum vouchers, photographs or recordings. No novel records came from e-vouchers, which contained 25 species on the Wiki Aves, and nine species on the Xeno-canto databases until 24/02/2017. The only species we failed to detect whose recent recording is available at those sites is the Rufous-breasted Leaftosser Sclerurus scansor
Table 2. Characteristics of seven localities with which the Ipanema National Forest was compared. Boraceia Biological Station (BBS), Barreiro Rico Farm (BRF), Carlos Botelho State Park (CBSP), Caetetus Ecological Station (CES), Intervales State Park (ISP), Lençóis Paulista (LP), Serra do Mar State Park (SMSP). Evidences are museum specimens $(\mathrm{S})$, photographs $(\mathrm{P})$ and sound recordings $(\mathrm{R})$.

\begin{tabular}{ccccccc}
\hline Habitat & $\begin{array}{c}\text { Elevation } \\
(\mathbf{m})\end{array}$ & $\begin{array}{c}\text { Area } \\
(\mathbf{h a})\end{array}$ & $\begin{array}{c}\text { Surveys } \\
(\mathbf{y r})\end{array}$ & Evidence & $\begin{array}{c}\text { Species } \\
\text { richness }\end{array}$ \\
\hline $\mathrm{BBS}$ & $\begin{array}{c}\text { Ombrophylous } \\
\text { forest }\end{array}$ & 800 & 96 & $1945-2010$ & $\mathrm{~S}, \mathrm{P}, \mathrm{R}$ & 323 \\
$\mathrm{BRF}$ & $\begin{array}{c}\text { Semidecidous } \\
\text { forest }\end{array}$ & $450-586$ & 1451 & $1957-2002$ & $\mathrm{~S}, \mathrm{P}, \mathrm{R}$ & 359 \\
$\mathrm{CBSP}$ & $\begin{array}{c}\text { Ombrophylous } \\
\text { forest }\end{array}$ & $20-1000$ & 37,644 & $2006-2009$ & $\mathrm{~S}, \mathrm{P}, \mathrm{R}$ & 331 \\
$\mathrm{CES}$ & $\begin{array}{c}\text { Semidecidous } \\
\text { forest }\end{array}$ & $500-680$ & 2,180 & $1976-2006$ & $\mathrm{P}, \mathrm{R}$ & 293 \\
$\mathrm{ISP}$ & $\begin{array}{c}\text { Ombrophylous } \\
\text { forest }\end{array}$ & $60-1010$ & 38,000 & $1988-2015$ & $\mathrm{~S}, \mathrm{P}, \mathrm{R}$ & 338 \\
$\mathrm{LP}$ & $\begin{array}{c}\text { Semidecidous } \\
\text { forest }\end{array}$ & 570 & 1,600 & $1989-2002$ & $\mathrm{P}, \mathrm{R}$ & 300 \\
$\mathrm{SMSP}$ & $\begin{array}{c}\text { Ombrophylous } \\
\text { forest }\end{array}$ & $0-1200$ & 300,000 & $1898-2012$ & $\mathrm{~S}, \mathrm{P}, \mathrm{R}$ & 417 \\
\hline
\end{tabular}

(Ménétriès 1835). Of the 69 Atlantic Forest endemic species, 40 have no recent records; of the three Cerrado endemic species, two have no recent records; five, of all species, are under threat categories. One is threatened at the global level and one is critically endangered in Brazil (Table 3).

We show a cluster analysis of the bird community similarity in Figure 3. There are two distinct groups composed of (1) rain forests (Serra do Mar State Park, Boraceia Biological Station, Intervales and Carlos Botelho State Parks) and (2) semideciduous forests (Barreiro Rico Farm, Caetetus Ecological Station and Lençóis Paulista). When accounting for historical bird records, INF (INF1 in Figure 3) is most similar to the first rain forests cluster. However, when we incorporated only recent bird community composition (INF2 in Figure 3), INF nested within semideciduous forests. Dissimilarity indexes showed the same pattern (Table 4).

\section{Discussion}

\section{Changes in species richness}

Overall, 410 species of birds are reported for the INF, but 89 forest species and another 16 typical Cerrado/wet grassland species may no longer occur there. Of the 202 species recorded both during the nineteenth century and recent surveys, most resident species are widespread in the Atlantic Forest (Del Hoyo et al. 1992-2011). Approximately $60 \%$ of the bird community of the INF is still found. Of this, 87 (43\%) are forest species. However, another $72(36 \%)$ forest species collected by Natterer seem absent, i.e. we did not detect $45 \%$ of forest species over the last 29 years.

Our multivariate analyses suggest modifications resulting from severe forest loss as INF changed its bird species richness from one closely related to rain forests running parallel to the coastline to another, which resembles drier semideciduous forests of the interior. The savannization of moist forests in Colombia has been previously suggested due to deforestation and land practices (Cavelier et al. 1998). This suggestion linked human induced-fire damaging soils dating as far as Pre-Columbian times. The 200 years span shown in this paper may prove to drive similar results in the Atlantic Forest vegetation.

\subsection{Vegetation}

Part of the remarkable historical species richness is also due to a Cerrado (tropical savanna) enclave, which increased substantially the diversity of the bird community with typical Cerrado species, such as 


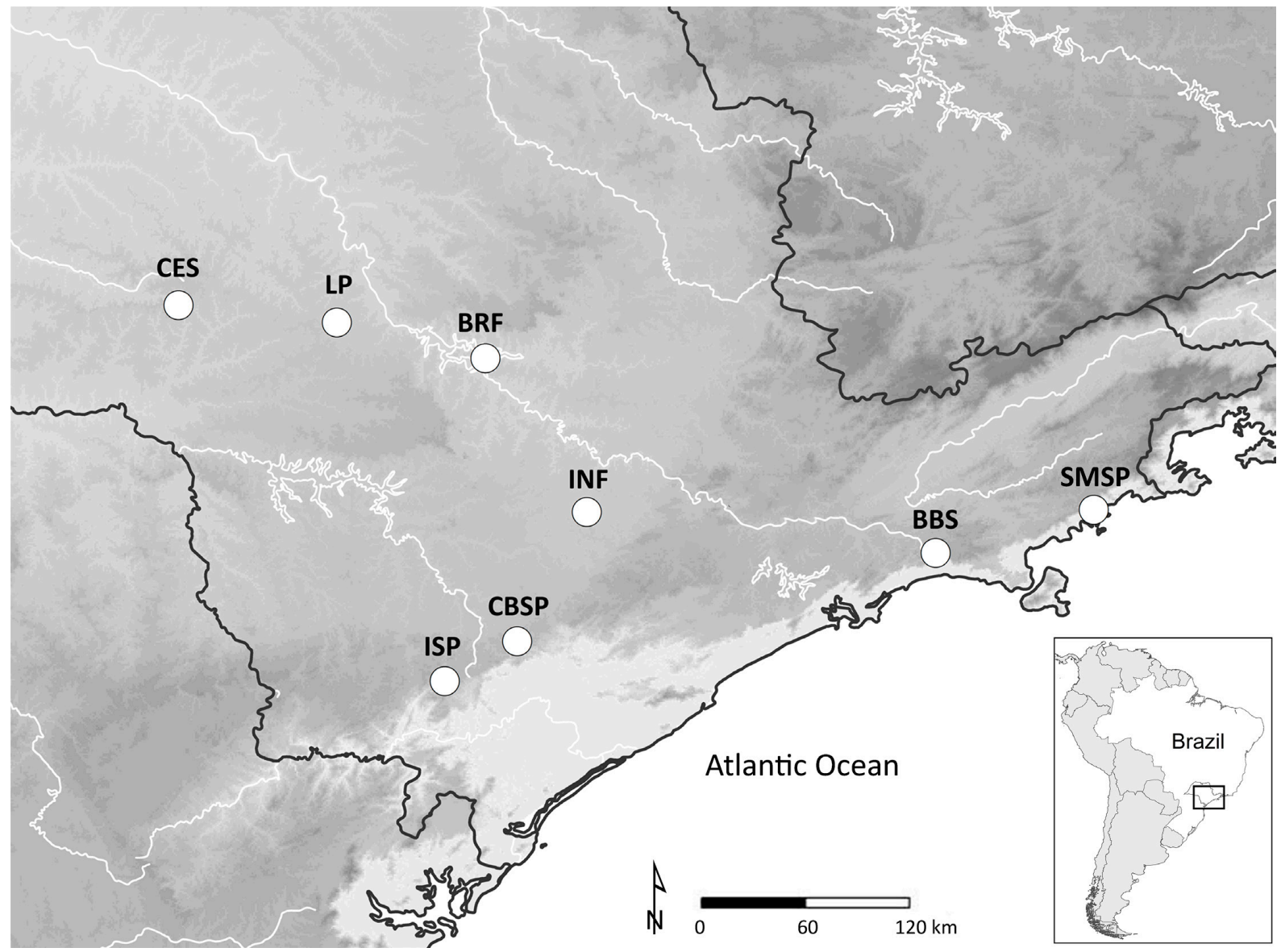

Figure 2. Locations between which we compared bird species richness. BBS - Boraceia Biological Station, CES - Caetetus Ecological Station, BRF - Bacury Farm, INF - Ipanema National Forest, LP - Lençóis Paulista, CBSP - Carlos Botelho State Park, ISP - Intervales State Park, SMSP - Serra do Mar State Park.

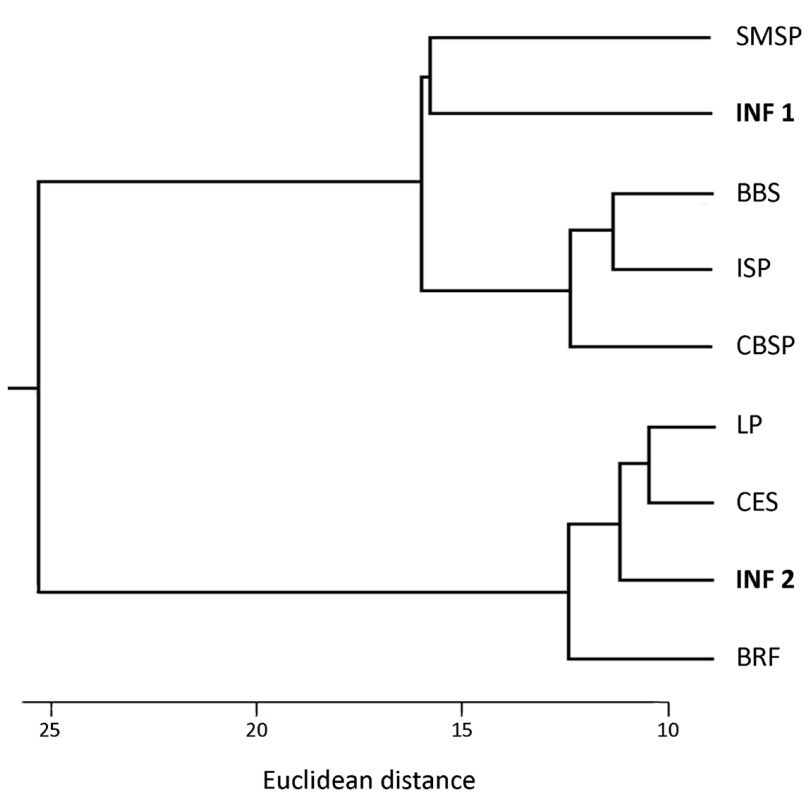

Figure 3. Cluster analysis of bird species richness similarity between eight Atlantic Forest remnants in São Paulo, southeastern Brazil. BBS - Boraceia Biological Station, CES - Caetetus Ecological Station, BRF - Bacury Farm, INF - Ipanema National Forest, LP - Lençóis Paulista, CBSP - Carlos Botelho State Park, ISP - Intervales State Park, SMSP - Serra do Mar State Park. INF 1 refers to historical species richness, whereas INF 2 refers to recent species richness.
Geositta poeciloptera (Wied 1830), Alectrurus tricolor (Vieillot 1816), Cistothorus platensis (Latham 1790) and Coryphaspiza melanotis (Temminck 1822). Although this enclave still exists, these species seem no longer present. Currently, no more than 29 Atlantic Forest and one Cerrado endemic species are detected in this area.

\subsection{Fragmentation}

The INF, where pristine forests once contained as much as 340 species of birds, currently has ca. 50\% of forest birds and several (69) invaders, i.e. species that did not occur at the INF and benefited from several years of deforestation (Table 3). Most species demanding high-quality forests or that are sensitive to human presence seem absent. These include Tinamus solitarius (Vieillot 1819), Aburria jacutinga (Spix 1825), Spizaetus ornatus (Daudin 1800), Triclaria malachitacea (Spix 1824), Trogon spp., Ramphastos spp. and Pteroglossus aracari (Linnaeus 1758), Helmeted Woodpecker Celeus galeatus (Temminck 1822), Short-tailed Antthrush Chamaeza campanisona (Lichtenstein 1823), Antpittas Grallaria varia (Boddaert 1783) and Hylopezus nattereri (Pinto 1937). Ipanema is the type locality of Phylloscartes eximius (Temminck 1822), a species without recent records. It is a sensitive mid-story flycatcher which requires riverine forests and occurs at low densities (Tonetti \& Pizo 2016). The species richness decline witnessed in the Psittaciformes and Trogoniformes is quite remarkable. Currently, one species of forest-dwelling parrot Pionus maximiliani (Kuhl 1820) is infrequent, and no Trogon sp. species remains. Because these species play essential ecological roles 
Cavarzere, V. et al.

Table 3. List of bird species (indicating those we consider forest dwellers) recorded within the Ipanema National Forest, São Paulo, southeastern Brazil. Sources: 1 (Pelzeln 1868), 2 (Reinhardt 1870), 3 (Hellmayr 1906), 4 (Stresemann 1948), 5 (Krabbe 2007), 6 (Silva \& Regalado 1998 ), 7 (Regalado 1999), 8 (Willis \& Oniki 2003), 9 (Silva 2010), 10 (present authors), WA (Wiki Aves), XC (Xeno-canto). Atlantic Forest (AF) and Cerrado (CE) endemic species are according to Parker et al. (1996) and Silva \& Bates (2002), respectively. Evidences acquired in this study are aural (A) or visual (V) undocumented records, or photographed (P), audio-recorded (R) or mist-netted (MN). Skins collected during the nineteenth century (sources 1-5) represent museum vouchers. A question mark denotes a probable misidentification or typographic error (see Lopes et al. 2017). Threat status: Critically Endangered (CR), Endangered (EN), Vulnerable $(\mathrm{VU})$. threatened in Brazil; SP = taxa threatened is São Paulo. Invader species are indicated as ${ }^{\text {inv }}$ on the recent records column.

\begin{tabular}{|c|c|c|c|c|c|c|}
\hline \multirow{2}{*}{ Taxon } & \multicolumn{2}{|c|}{ Record } & \multirow{2}{*}{ Endemism } & \multirow{2}{*}{ Evidence } & \multirow{2}{*}{ Threat status } & \multirow{2}{*}{ Habitat } \\
\hline & Historic & Recent & & & & \\
\hline \multicolumn{7}{|l|}{ Tinamiformes } \\
\hline \multicolumn{7}{|l|}{ Tinamidae } \\
\hline Tinamus solitarius & 1 & & $\mathrm{AF}$ & & & Forest \\
\hline Crypturellus obsoletus & 1 & & & & & Forest \\
\hline Crypturellus parvirostris & 1 & 8,10 & & A & & \\
\hline Crypturellus tataupa & 1 & 10 & & $\mathrm{~A}$ & & Forest \\
\hline Rhynchotus rufescens & 1,3 & & & & & \\
\hline Nothura maculosa & 1 & 8,10 & & $\mathrm{P}$ & & \\
\hline \multicolumn{7}{|l|}{ Anseriformes } \\
\hline \multicolumn{7}{|l|}{ Anhimidae } \\
\hline Anhima cornuta & 1 & & & & & \\
\hline \multicolumn{7}{|l|}{ Anatidae } \\
\hline Dendrocygna viduata & & $10^{\text {inv }}$ & & $\mathrm{V}$ & & \\
\hline Cairina moschata & 1 & 10 & & $\mathrm{P}$ & & \\
\hline Amazonetta brasiliensis & 1 & 9,10 & & $\mathrm{~V}$ & & \\
\hline Nomonyx dominicus & 1 & & & & & \\
\hline \multicolumn{7}{|l|}{ Galliformes } \\
\hline \multicolumn{7}{|l|}{ Cracidae } \\
\hline Penelope superciliaris & 1 & 9,10 & & $\mathrm{P}$ & & Forest \\
\hline Aburria jacutinga & 1 & & $\mathrm{AF}$ & & $\mathrm{EN}^{\mathrm{BR}}$ & Forest \\
\hline \multicolumn{7}{|l|}{ Odontophoridae } \\
\hline Odontophorus capueira & 1 & & $\mathrm{AF}$ & & & Forest \\
\hline \multicolumn{7}{|l|}{ Podicipediformes } \\
\hline \multicolumn{7}{|l|}{ Podicipedidae } \\
\hline Tachybaptus dominicus & 1,3 & 10 & & $\mathrm{R}, \mathrm{P}$ & & \\
\hline Podilymbus podiceps & 1 & 9,10 & & $\mathrm{~V}$ & & \\
\hline \multicolumn{7}{|l|}{ Suliformes } \\
\hline \multicolumn{7}{|l|}{ Phalacrocoracidae } \\
\hline Nannopterum brasilianus & 1 & 9,10 & & $\mathrm{~V}$ & & \\
\hline Anhingidae & & & & & & \\
\hline Anhinga anhinga & & $9,10^{\text {inv }}$ & & $\mathrm{V}$ & & \\
\hline Pelecaniformes & & & & & & \\
\hline Ardeidae & & & & & & \\
\hline Tigrisoma lineatum & 1 & 10 & & $\mathrm{~V}$ & & \\
\hline Ixobrychus exilis & 1 & 8 & & & & \\
\hline Nycticorax nycticorax & 1 & 9,10 & & $\mathrm{~V}$ & & \\
\hline Butorides striata & 1 & 9,10 & & $\mathrm{~V}$ & & \\
\hline Bubulcus ibis & & $9,10^{\text {inv }}$ & & $\mathrm{V}$ & & \\
\hline Ardea cocoi & 1 & 9,10 & & $\mathrm{~V}$ & & \\
\hline Ardea alba & 1 & 9,10 & & $\mathrm{~V}$ & & \\
\hline Syrigma sibilatrix & & $9,10^{\text {inv }}$ & & $\mathrm{V}$ & & \\
\hline Pilherodius pileatus & 1 & & & & & \\
\hline Egretta thula & 1 & 9,10 & & $\mathrm{~V}$ & & \\
\hline Egretta caerulea & 1 & & & & & \\
\hline Threskiornithidae & & & & & & \\
\hline Plegadis chihi & 1 & & & & & \\
\hline Mesembrinibis cayennensis & 1 & 10 & & $\mathrm{~V}$ & & \\
\hline Platalea ajaja & 1 & & & & & \\
\hline Cathartiformes & & & & & & \\
\hline Cathartidae & & & & & & \\
\hline Cathartes aura & 1 & 9,10 & & $\mathrm{P}$ & & \\
\hline Coragyps atratus & 1 & $8,9,10$ & & $\mathrm{P}$ & & \\
\hline Sarcoramphus papa & & $6,9,10^{\text {inv }}$ & & $\mathrm{P}$ & $\mathrm{EN}^{\mathrm{SP}}$ & \\
\hline
\end{tabular}


Table 3. Continued...

\begin{tabular}{|c|c|c|c|c|c|c|}
\hline \multirow{2}{*}{ Taxon } & \multicolumn{2}{|c|}{ Record } & \multirow{2}{*}{ Endemism } & \multirow{2}{*}{ Evidence } & \multirow{2}{*}{ Threat status } & \multirow{2}{*}{ Habitat } \\
\hline & Historic & Recent & & & & \\
\hline \multicolumn{7}{|l|}{ Accipitriformes } \\
\hline \multicolumn{7}{|l|}{ Accipitridae } \\
\hline Leptodon cayanensis & 1 & 10 & & $\mathrm{P}$ & & Forest \\
\hline Elanoides forficatus & 1 & & & & & \\
\hline Elanus leucurus & & $8,9,10^{\text {inv }}$ & & $\mathrm{P}$ & & \\
\hline Harpagus diodon & 1 & 10 & & $\mathrm{~V}$ & & Forest \\
\hline Accipiter poliogaster & 1 & & & & & \\
\hline Accipiter superciliosus & 1 & & & & & \\
\hline Ictinia plumbea & 1 & 9,10 & & $\mathrm{P}$ & & \\
\hline Rostrhamus sociabilis & & $9,10^{\text {inv }}$ & & $\mathrm{V}$ & & \\
\hline Heterospizias meridionalis & 1 & 9,10 & & V & & \\
\hline Urubitinga urubitinga & & $10^{\text {inv }}$ & & $\mathrm{V}$ & & \\
\hline Urubitinga coronata & & $9^{\text {inv }}$ & & $\mathrm{V}$ & $\mathrm{EN}^{\mathrm{IUCN}}$ & \\
\hline Rupornis magnirostris & 1 & 9,10 & & $\mathrm{P}$ & & \\
\hline Geranoaetus albicaudatus & 1 & 9,10 & & $\mathrm{~V}$ & & \\
\hline Pseudastur polionotus & 1 & & $\mathrm{AF}$ & & & Forest \\
\hline Buteo brachyurus & & $9^{\text {inv }}$ & & $\mathrm{V}$ & & \\
\hline Spizaetus tyrannus & 1,3 & & & & & Forest \\
\hline Spizaetus melanoleucus & 1 & & & & & \\
\hline Spizaetus ornatus & 1 & & & & & Forest \\
\hline \multicolumn{7}{|l|}{ Gruiformes } \\
\hline \multicolumn{7}{|l|}{ Aramidae } \\
\hline Aramus guarauna & & $9,10^{\text {inv }}$ & & $\mathrm{V}$ & & \\
\hline \multicolumn{7}{|l|}{ Rallidae } \\
\hline Aramides cajaneus & & $9,10^{\text {inv }}$ & & $\mathrm{V}$ & & \\
\hline Aramides saracura & 1 & 10 & $\mathrm{AF}$ & $\mathrm{P}$ & & Forest \\
\hline Laterallus melanophaius & & $10^{\text {inv }}$ & & A & & \\
\hline Mustelirallus albicollis & 1 & 9 & & & & \\
\hline Pardirallus nigricans & 1 & 9,10 & & A & & \\
\hline Gallinula galeata & 1 & $8,9,10$ & & $\mathrm{P}$ & & \\
\hline Porphyrio martinicus & 1 & 9 & & & & \\
\hline \multicolumn{7}{|l|}{ Charadriiformes } \\
\hline \multicolumn{7}{|l|}{ Charadriidae } \\
\hline Vanellus chilensis & 1 & $8,9,10$ & & $\mathrm{~A}, \mathrm{~V}$ & & \\
\hline Pluvialis dominica & 1 & & & & & \\
\hline \multicolumn{7}{|l|}{ Recurvirostridae } \\
\hline Himantopus melanurus & 1 & & & & & \\
\hline \multicolumn{7}{|l|}{ Scolopacidae } \\
\hline Gallinago paraguaiae & 1 & & & & & \\
\hline Gallinago undulata & 1 & & & & & \\
\hline Numenius borealis & 1 & & & & & \\
\hline Actitis macularius & 1 & & & & & \\
\hline Tringa solitaria & 1 & & & & & \\
\hline Tring a flavipes & 1 & & & & & \\
\hline Calidris alba & 1 & & & & & \\
\hline Calidris fuscicollis & 1 & & & & & \\
\hline Calidris melanotos & 1 & & & & & \\
\hline Calidris subruficollis & 1 & & & & & \\
\hline \multicolumn{7}{|l|}{ Jacanidae } \\
\hline Jacana jacana & 1 & $8,9,10$ & & $\mathrm{~A}, \mathrm{~V}$ & & \\
\hline \multicolumn{7}{|l|}{ Laridae } \\
\hline Chroicocephalus maculipennis & 1 & & & & & \\
\hline Sternidae & & & & & & \\
\hline Sternula superciliaris & 1 & & & & & \\
\hline Rynchopidae & & & & & & \\
\hline Rynchops niger & 1 & & & & & \\
\hline
\end{tabular}


Table 3. Continued..

\begin{tabular}{|c|c|c|c|c|c|c|}
\hline \multirow{2}{*}{ Taxon } & \multicolumn{2}{|c|}{ Record } & \multirow{2}{*}{ Endemism } & \multirow{2}{*}{ Evidence } & \multirow{2}{*}{ Threat status } & \multirow{2}{*}{ Habitat } \\
\hline & Historic & Recent & & & & \\
\hline \multicolumn{7}{|l|}{ Columbiformes } \\
\hline \multicolumn{7}{|l|}{ Columbidae } \\
\hline Columbina talpacoti & & $8,9,10^{\text {inv }}$ & & $\mathrm{A}, \mathrm{V}$ & & \\
\hline Columbina squammata & & $9,10^{\text {inv }}$ & & $\mathrm{A}, \mathrm{V}$ & & \\
\hline Claravis pretiosa & 1 & & & & & Forest \\
\hline Claravis geoffroyi & 1 & & $\mathrm{AF}$ & & $\mathrm{CR}^{\mathrm{BR}}$ & Forest \\
\hline Columba livia & & $9^{\text {inv }}$ & & $\mathrm{V}$ & & \\
\hline Patagioenas picazuro & & $9,10^{\text {inv }}$ & & $\mathrm{P}$ & & \\
\hline Patagioenas cayennensis & 1 & 9,10 & & $\mathrm{~A}, \mathrm{~V}$ & & Forest \\
\hline Zenaida auriculata & 1 & $8,9,10$ & & $\mathrm{~A}, \mathrm{~V}$ & & \\
\hline Leptotila verreauxi & 1 & 9,10 & & $\mathrm{MN}, \mathrm{P}$ & & Forest \\
\hline Leptotila rufaxilla & 1 & 9,10 & & A & & Forest \\
\hline Geotrygon violacea & 1 & & & & & Forest \\
\hline Geotrygon montana & 1 & 10 & & A & & Forest \\
\hline \multicolumn{7}{|l|}{ Cuculiformes } \\
\hline \multicolumn{7}{|l|}{ Cuculidae } \\
\hline Piaya cayana & 1 & 9,10 & & $\mathrm{P}$ & & \\
\hline Coccyzus melacoryphus & 1 & & & & & \\
\hline Coccyzus euleri & & $10^{\text {inv }}$ & & $\mathrm{P}$ & & \\
\hline Crotophaga ani & 1 & $8,9,10$ & & $\mathrm{P}$ & & \\
\hline Guira guira & 1 & $8,9,10$ & & $\mathrm{~A}, \mathrm{~V}$ & & \\
\hline Tapera naevia & 1 & 9,10 & & $\mathrm{R}, \mathrm{P}$ & & Forest \\
\hline Dromococcyx phasianellus & 1 & & & & & Forest \\
\hline Dromococcyx pavoninus & & $10^{\text {inv }}$ & & A & & \\
\hline \multicolumn{7}{|l|}{ Strigiformes } \\
\hline \multicolumn{7}{|l|}{ Tytonidae } \\
\hline Tyto furcata & 1 & 10 & & $\mathrm{~V}$ & & \\
\hline \multicolumn{7}{|l|}{ Strigidae } \\
\hline Megascops choliba & 1 & 9,10 & & A & & Forest \\
\hline Pulsatrix koeniswaldiana & 1 & 10 & $\mathrm{AF}$ & A & & Forest \\
\hline Strix hylophila & 1 & & $\mathrm{AF}$ & & & Forest \\
\hline Strix virgata & 1 & & & & & Forest \\
\hline Glaucidium brasilianum & 1 & & & & & \\
\hline Athene cunicularia & & $8,9,10^{\text {inv }}$ & & $\mathrm{A}, \mathrm{V}$ & & \\
\hline Asio clamator & 1 & & & & & \\
\hline Asio flammeus & 1 & & & & & \\
\hline \multicolumn{7}{|l|}{ Caprimulgiformes } \\
\hline \multicolumn{7}{|l|}{ Nyctibiidae } \\
\hline Nyctibius aethereus & 1 & & & & $\mathrm{EN}^{\mathrm{BR}}$ & Forest \\
\hline Nyctibius griseus & 1 & 9,10 & & A & & Forest \\
\hline \multicolumn{7}{|l|}{ Caprimulgidae } \\
\hline Nyctiphrynus ocellatus & 1 & & & & & Forest \\
\hline Antrostomus rufus & & $9,10^{\text {inv }}$ & & $\mathrm{R}$ & & \\
\hline Antrostomus sericocaudatus & 1 & & & & & Forest \\
\hline Lurocalis semitorquatus & 1,4 & 10 & & $\mathrm{~A}, \mathrm{~V}$ & & Forest \\
\hline Nyctidromus albicollis & 1 & 9,10 & & $\mathrm{~A}, \mathrm{~V}$ & & \\
\hline Hydropsalis parvula & 1 & 10 & & $\mathrm{~A}, \mathrm{~V}$ & & \\
\hline Hydropsalis anomala & 1 & & & & & \\
\hline Hydropsalis maculicaudus & 1 & & & & & \\
\hline Hydropsalis torquata & 1 & 9,10 & & $\mathrm{P}$ & & \\
\hline Hydropsalis forcipata & 1 & & $\mathrm{AF}$ & & & \\
\hline Podager nacunda & 1,4 & 10 & & $\mathrm{~V}$ & & \\
\hline Chordeiles minor & 1 & & & & & \\
\hline
\end{tabular}


Table 3. Continued...

\begin{tabular}{|c|c|c|c|c|c|c|}
\hline \multirow{2}{*}{ Taxon } & \multicolumn{2}{|c|}{ Record } & \multirow{2}{*}{ Endemism } & \multirow{2}{*}{ Evidence } & \multirow{2}{*}{ Threat status } & \multirow{2}{*}{ Habitat } \\
\hline & Historic & Recent & & & & \\
\hline \multicolumn{7}{|l|}{ Apodiformes } \\
\hline \multicolumn{7}{|l|}{ Apodidae } \\
\hline Cypseloides fumigatus & 1 & & & & & \\
\hline Cypseloides senex & 1 & & & & & \\
\hline Streptoprocne zonaris & 1 & 9,10 & & $\mathrm{~V}$ & & \\
\hline Streptoprocne biscutata & 1 & & & & & \\
\hline Chaetura meridionalis & 1 & $8,9,10$ & & $\mathrm{~A}, \mathrm{~V}$ & & \\
\hline \multicolumn{7}{|l|}{ Trochilidae } \\
\hline Phaethornis squalidus & 1 & & $\mathrm{AF}$ & & & \\
\hline Phaethornis pretrei & & $9,10^{\text {inv }}$ & & $\mathrm{A}, \mathrm{V}$ & & \\
\hline Phaethornis eurynome & 1 & 9,10 & $\mathrm{AF}$ & $\mathrm{A}, \mathrm{V}$ & & Forest \\
\hline Campylopterus largipennis & $1 ?$ & & & & & \\
\hline Eupetomena macroura & & $9,10^{\text {inv }}$ & & $P$ & & \\
\hline Aphantochroa cirrochloris & 1 & & & & & Forest \\
\hline Florisuga fusca & 1 & 9,10 & & $\mathrm{~A}, \mathrm{~V}$ & & \\
\hline Colibri serrirostris & 1 & 8,10 & & $\mathrm{P}$ & & \\
\hline Anthracothorax nigricollis & 1 & 9 & & & & \\
\hline Lophornis magnificus & 1 & & & & & Forest \\
\hline Lophornis chalybeus & 1,3 & & & & & Forest \\
\hline Chlorostilbon lucidus & 1 & 9,10 & & $\mathrm{P}$ & & \\
\hline Thalurania glaucopis & 1 & 9,10 & $\mathrm{AF}$ & MN,P & & Forest \\
\hline Leucochloris albicollis & 1 & 9,10 & & $\mathrm{P}$ & & Forest \\
\hline Amazilia versicolor & 1 & 10 & & $\mathrm{~A}, \mathrm{~V}$ & & Forest \\
\hline Amazilia lactea & & $9,10^{\text {inv }}$ & & $\mathrm{A}, \mathrm{V}$ & & \\
\hline Heliodoxa rubricauda & 1 & & $\mathrm{AF}$ & & & Forest \\
\hline Heliothryx auritus & 1 & & & & & \\
\hline Heliomaster squamosus & 1 & 10 & & $\mathrm{P}$ & & \\
\hline Calliphlox amethystina & 1 & & & & & \\
\hline \multicolumn{7}{|l|}{ Trogoniformes } \\
\hline \multicolumn{7}{|l|}{ Trogonidae } \\
\hline Trogon surrucura & 1 & & & & & Forest \\
\hline Trogon rufus & 1 & & & & & Forest \\
\hline \multicolumn{7}{|l|}{ Coraciiformes } \\
\hline \multicolumn{7}{|l|}{ Alcedinidae } \\
\hline Megaceryle torquata & 1 & 9,10 & & $\mathrm{~A}, \mathrm{~V}$ & & \\
\hline Chloroceryle amazona & 1 & $8,9,10$ & & V & & \\
\hline Chloroceryle americana & 1 & 9,10 & & $\mathrm{~V}$ & & \\
\hline \multicolumn{7}{|l|}{ Momotidae } \\
\hline Baryphthengus ruficapillus & 1 & 10 & & $\mathrm{~A}, \mathrm{~V}$ & & Forest \\
\hline \multicolumn{7}{|l|}{ Galbuliformes } \\
\hline \multicolumn{7}{|l|}{ Galbulidae } \\
\hline Jacamaralcyon tridactyla & 1,3 & & $\mathrm{AF}$ & & & \\
\hline \multicolumn{7}{|l|}{ Bucconidae } \\
\hline Notharchus swainsoni & 1 & & $\mathrm{AF}$ & & & Forest \\
\hline Nystalus chacuru & 1 & 9 & & & & \\
\hline Malacoptila striata & 1 & 9,10 & & MN,P & & Forest \\
\hline Nonnula rubecula & 1 & & & & & Forest \\
\hline \multicolumn{7}{|l|}{ Piciformes } \\
\hline \multicolumn{7}{|l|}{ Ramphastidae } \\
\hline Ramphastos toco & 1 & 9,10 & & V & & \\
\hline Ramphastos dicolorus & 1 & & $\mathrm{AF}$ & & & Forest \\
\hline Pteroglossus aracari & 1 & & & & & Forest \\
\hline
\end{tabular}


Cavarzere, V. et al.

Table 3. Continued..

\begin{tabular}{|c|c|c|c|c|c|c|}
\hline \multirow{2}{*}{ Taxon } & \multicolumn{2}{|c|}{ Record } & \multirow{2}{*}{ Endemism } & \multirow{2}{*}{ Evidence } & \multirow{2}{*}{ Threat status } & \multirow{2}{*}{ Habitat } \\
\hline & Historic & Recent & & & & \\
\hline \multicolumn{7}{|l|}{ Picidae } \\
\hline Picumnus cirratus & 1 & 9,10 & & $\mathrm{~A}, \mathrm{~V}$ & & Forest \\
\hline Picumnus temminckii & 1 & 9,10 & $\mathrm{AF}$ & $\mathrm{A}, \mathrm{V}$ & & Forest \\
\hline Melanerpes candidus & 1 & $8,9,10$ & & $\mathrm{~A}, \mathrm{~V}$ & & \\
\hline Melanerpes flavifrons & 1 & & & & & Forest \\
\hline Veniliornis spilogaster & 1 & 9,10 & & $\mathrm{~A}, \mathrm{~V}$ & & Forest \\
\hline Piculus aurulentus & 1 & & $\mathrm{AF}$ & & & Forest \\
\hline Colaptes melanochloros & 1 & 9,10 & & $\mathrm{R}$ & & \\
\hline Colaptes campestris & 1 & $8,9,10$ & & $\mathrm{~A}, \mathrm{~V}$ & & \\
\hline Celeus galeatus & 1 & & $\mathrm{AF}$ & & $\mathrm{EN}^{\mathrm{BR}}$ & Forest \\
\hline Celeus flavescens & 1 & 9,10 & & $\mathrm{~A}, \mathrm{~V}$ & & Forest \\
\hline Dryocopus lineatus & 1 & 9,10 & & $\mathrm{P}$ & & \\
\hline Campephilus robustus & 1 & & $\mathrm{AF}$ & & & Forest \\
\hline \multicolumn{7}{|l|}{ Cariamiformes } \\
\hline \multicolumn{7}{|l|}{ Cariamidae } \\
\hline Cariama cristata & & $9,10^{\text {inv }}$ & & $\mathrm{A}, \mathrm{V}$ & & \\
\hline \multicolumn{7}{|l|}{ Falconiformes } \\
\hline \multicolumn{7}{|l|}{ Falconidae } \\
\hline Ibycter americanus & 1 & & & & & \\
\hline Caracara plancus & 1 & 9,10 & & $\mathrm{P}$ & & \\
\hline Milvago chimachima & 1,3 & 9,10 & & $\mathrm{~V}$ & & \\
\hline Herpetotheres cachinnans & 9 & 10 & & A & & \\
\hline Micrastur ruficollis & 1 & & & & & Forest \\
\hline Micrastur semitorquatus & & $10^{\text {inv }}$ & & A & & \\
\hline Falco sparverius & 1 & 9,10 & & $\mathrm{~V}$ & & \\
\hline Falco femoralis & 1 & 9,10 & & $\mathrm{~V}$ & & \\
\hline \multicolumn{7}{|l|}{ Psittaciformes } \\
\hline \multicolumn{7}{|l|}{ Psittacidae } \\
\hline Primolius maracana & 1 & & & & & Forest \\
\hline Psittacara leucophthalmus & 1 & 10 & & $\mathrm{~A}, \mathrm{~V}$ & & \\
\hline Aratinga auricapillus & 1 & 10 & & $\mathrm{R}$ & & \\
\hline Pyrrhura frontalis & 1 & & & & & Forest \\
\hline Forpus xanthopterygius & 1 & 9,10 & & $\mathrm{~A}, \mathrm{~V}$ & & \\
\hline Brotogeris tirica & & $10^{\text {inv }}$ & $\mathrm{AF}$ & $\mathrm{A}, \mathrm{V}$ & & \\
\hline Pionopsitta pileata & 1 & & $\mathrm{AF}$ & & & Forest \\
\hline Pionus maximiliani & 1 & 9,10 & & $\mathrm{~A}, \mathrm{~V}$ & & Forest \\
\hline Amazona vinacea & 1,3 & & $\mathrm{AF}$ & & $\mathrm{VU}^{\mathrm{BR}}$ & Forest \\
\hline Amazona aestiva & & $10^{\text {inv }}$ & & $\mathrm{A}, \mathrm{V}$ & & \\
\hline Triclaria malachitacea & 1 & & $\mathrm{AF}$ & & & Forest \\
\hline \multicolumn{7}{|l|}{ Passeriformes } \\
\hline \multicolumn{7}{|l|}{ Thamnophilidae } \\
\hline Rhopias gularis & 1 & & & & & Forest \\
\hline Dysithamnus mentalis & & $9,10^{\text {inv }}$ & & $\mathrm{A}, \mathrm{MN}, \mathrm{V}$ & & \\
\hline Herpsilochmus rufimarginatus & 1 & & & & & Forest \\
\hline Thamnophilus doliatus & & $10^{\text {inv }}$ & & $\mathrm{P}$ & & \\
\hline Thamnophilus ruficapillus & 1 & 10 & & $\mathrm{R}$ & & \\
\hline Thamnophilus caerulescens & 1 & $7,9,10$ & & $\mathrm{~A}, \mathrm{MN}, \mathrm{V}$ & & Forest \\
\hline Hypoedaleus guttatus & 1,3 & & $\mathrm{AF}$ & & & Forest \\
\hline Batara cinerea & 1 & & & & & Forest \\
\hline Mackenziaena leachii & 1 & & $\mathrm{AF}$ & & & Forest \\
\hline Mackenziaena severa & 1 & $7,9,10$ & $\mathrm{AF}$ & $\mathrm{A}, \mathrm{MN}, \mathrm{V}$ & & Forest \\
\hline Biatas nigropectus & 1 & & $\mathrm{AF}$ & & & Forest \\
\hline Myrmoderus squamosus & 1 & 10 & $\mathrm{AF}$ & A & & Forest \\
\hline Pyriglena leucoptera & 1 & $7,9,10$ & $\mathrm{AF}$ & $\mathrm{A}, \mathrm{MN}, \mathrm{V}$ & & Forest \\
\hline Drymophila ferruginea & 1 & & $\mathrm{AF}$ & & & Forest \\
\hline Drymophila ochropyga & 1 & & $\mathrm{AF}$ & & & Forest \\
\hline Drymophila malura & 1 & $7,9,10$ & $\mathrm{AF}$ & $\mathrm{R}$ & & Forest \\
\hline
\end{tabular}


Table 3. Continued...

\begin{tabular}{|c|c|c|c|c|c|c|}
\hline \multirow{2}{*}{ Taxon } & \multicolumn{2}{|c|}{ Record } & \multirow{2}{*}{ Endemism } & \multirow{2}{*}{ Evidence } & \multirow{2}{*}{ Threat status } & \multirow{2}{*}{ Habitat } \\
\hline & Historic & Recent & & & & \\
\hline \multicolumn{7}{|l|}{ Conopophagidae } \\
\hline Conopophaga lineata & 1 & $7,9,10$ & & $\mathrm{~A}, \mathrm{MN}, \mathrm{V}$ & & Forest \\
\hline \multicolumn{7}{|l|}{ Grallariidae } \\
\hline Grallaria varia & 1 & & & & & Forest \\
\hline Hylopezus nattereri & 1 & & $\mathrm{AF}$ & & & Forest \\
\hline \multicolumn{7}{|l|}{ Rhinocryptidae } \\
\hline Eleoscytalopus indigoticus & 1 & 9,10 & $\mathrm{AF}$ & $\mathrm{A}$ & & Forest \\
\hline \multicolumn{7}{|l|}{ Formicariidae } \\
\hline Chamaeza campanisona & 1 & & & & & Forest \\
\hline \multicolumn{7}{|l|}{ Scleruridae } \\
\hline Sclerurus scansor & 1 & WA,XC & $\mathrm{AF}$ & $\mathrm{R}$ & & Forest \\
\hline Geositta poeciloptera & 1 & & $\mathrm{CE}$ & & $\mathrm{EN}^{\mathrm{BR}}$ & \\
\hline \multicolumn{7}{|l|}{ Dendrocolaptidae } \\
\hline Sittasomus griseicapillus & 1 & $7,9,10$ & & $\mathrm{~A}, \mathrm{MN}, \mathrm{V}$ & & Forest \\
\hline Xiphorhynchus fuscus & 1 & $7,9,10$ & & $\mathrm{~A}, \mathrm{~V}$ & & Forest \\
\hline Campylorhamphus falcularius & 1 & $7,9,10$ & $\mathrm{AF}$ & $\mathrm{A}, \mathrm{V}$ & & Forest \\
\hline Lepidocolaptes angustirostris & & $10^{\text {inv }}$ & & $\mathrm{V}$ & & \\
\hline Lepidocolaptes falcinellus & 1 & 10 & $\mathrm{AF}$ & $\mathrm{V}$ & & Forest \\
\hline Dendrocolaptes platyrostris & 1 & $7,9,10$ & & A & & Forest \\
\hline Xiphocolaptes albicollis & 1 & & & & & \\
\hline \multicolumn{7}{|l|}{ Furnariidae } \\
\hline Xenops minutus & 1 & & & & & Forest \\
\hline Xenops rutilans & 1 & 7,10 & & $\mathrm{~A}, \mathrm{~V}$ & & Forest \\
\hline Furnarius rufus & & $8,9,10^{\text {inv }}$ & & $\mathrm{A}, \mathrm{V}$ & & \\
\hline Lochmias nematura & 1 & $7,9,10$ & & $\mathrm{~A}, \mathrm{MN}$ & & Forest \\
\hline Automolus leucophthalmus & 1 & $7,9,10$ & & $\mathrm{~A}, \mathrm{MN}$ & & Forest \\
\hline Anabazenops fuscus & 1 & & $\mathrm{AF}$ & & & Forest \\
\hline Anabacerthia amaurotis & 1 & & $\mathrm{AF}$ & & & Forest \\
\hline Anabacerthia lichtensteini & 1 & & $\mathrm{AF}$ & & & Forest \\
\hline Philydor atricapillus & 1 & 8 & $\mathrm{AF}$ & & & Forest \\
\hline Philydor rufum & 1 & & & & & Forest \\
\hline Heliobletus contaminatus & 1 & 7,9 & $\mathrm{AF}$ & & & Forest \\
\hline Syndactyla rufosuperciliata & 1 & 9,10 & & $\mathrm{~A}, \mathrm{MN}, \mathrm{V}$ & & Forest \\
\hline Phacellodomus ferrugineigula & & $8,10^{\text {inv }}$ & $\mathrm{AF}$ & $\mathrm{P}$ & & \\
\hline Certhiaxis cinnamomeus & 1 & 9,10 & & $\mathrm{~A}$ & & \\
\hline Synallaxis ruficapilla & 1 & $7,9,10$ & $\mathrm{AF}$ & $\mathrm{A}, \mathrm{MN}, \mathrm{V}$ & & Forest \\
\hline Synallaxis cinerascens & 1 & & $\mathrm{AF}$ & & & Forest \\
\hline Synallaxis frontalis & & $9,10^{\text {inv }}$ & & $\mathrm{MN}, \mathrm{P}$ & & \\
\hline Synallaxis albescens & & $9^{\text {inv }}$ & & $\mathrm{A}$ & & \\
\hline Synallaxis spixi & 1 & 9,10 & & A & & \\
\hline Cranioleuca pallida & 1 & $7,9,10$ & $\mathrm{AF}$ & $\mathrm{A}$ & & Forest \\
\hline \multicolumn{7}{|l|}{ Pipridae } \\
\hline Neopelma chrysolophum & 1 & & $\mathrm{AF}$ & & & Forest \\
\hline Manacus manacus & 1 & & & & & Forest \\
\hline Ilicura militaris & 1 & & & & & Forest \\
\hline Chiroxiphia caudata & 1 & $7,9,10$ & $\mathrm{AF}$ & $\mathrm{A}, \mathrm{MN}, \mathrm{V}$ & & Forest \\
\hline \multicolumn{7}{|l|}{ Tityridae } \\
\hline Myiobius atricaudus & 1 & & & & & Forest \\
\hline Schiffornis virescens & 1,5 & 10 & $\mathrm{AF}$ & A & & Forest \\
\hline Laniisoma elegans & 1 & $\begin{array}{l}\text { M. Cohn-Haft and } \\
\text { A. Whittaker }\end{array}$ & & $\mathrm{R}$ & $\mathrm{VU}^{\mathrm{SP}}$ & Forest \\
\hline Tityra inquisitor & 1 & 9 & & & & Forest \\
\hline Tityra cayana & 1 & 7,9 & & & & Forest \\
\hline Pachyramphus viridis & 1 & 10 & & A & & Forest \\
\hline Pachyramphus castaneus & 1 & & & & & Forest \\
\hline Pachyramphus polychopterus & & $9,10^{\text {inv }}$ & & $\mathrm{A}, \mathrm{V}$ & & \\
\hline Pachyramphus validus & 1 & 9,10 & & $\mathrm{~A}, \mathrm{~V}$ & & \\
\hline
\end{tabular}


Cavarzere, V. et al.

Table 3. Continued...

\begin{tabular}{|c|c|c|c|c|c|c|}
\hline \multirow{2}{*}{ Taxon } & \multicolumn{2}{|c|}{ Record } & \multirow{2}{*}{ Endemism } & \multirow{2}{*}{ Evidence } & \multirow{2}{*}{ Threat status } & \multirow{2}{*}{ Habitat } \\
\hline & Historic & Recent & & & & \\
\hline \multicolumn{7}{|l|}{ Cotingidae } \\
\hline Pyroderus scutatus & 1 & 7 & & & $\mathrm{VU}^{\mathrm{SP}}$ & Forest \\
\hline Lipaugus lanioides & 1 & & $\mathrm{AF}$ & & & Forest \\
\hline Procnias nudicollis & 1 & 9 & $\mathrm{AF}$ & & $\mathrm{VU}^{\mathrm{SP}}$ & Forest \\
\hline \multicolumn{7}{|l|}{ Pipritidae } \\
\hline Piprites chloris & 1 & & & & & Forest \\
\hline \multicolumn{7}{|l|}{ Platyrinchidae } \\
\hline Platyrinchus mystaceus & 1,5 & $7,9,10$ & & $\mathrm{~A}, \mathrm{MN}, \mathrm{V}$ & & Forest \\
\hline \multicolumn{7}{|l|}{ Rhynchocyclidae } \\
\hline Mionectes rufiventris & 1 & $7,9,10$ & & $\mathrm{~A}, \mathrm{~V}$ & & Forest \\
\hline Leptopogon amaurocephalus & 1 & $7,9,10$ & & $\mathrm{~A}, \mathrm{MN}, \mathrm{V}$ & & Forest \\
\hline Corythopis delalandi & 1 & 10 & & $\mathrm{~A}, \mathrm{MN}, \mathrm{V}$ & & Forest \\
\hline Phylloscartes eximius & 1 & & $\mathrm{AF}$ & & & Forest \\
\hline Phylloscartes ventralis & 1 & 10 & & $\mathrm{R}$ & & Forest \\
\hline Tolmomyias sulphurescens & 1 & $7,9,10$ & & $\mathrm{~A}, \mathrm{~V}$ & & Forest \\
\hline Todirostrum poliocephalum & & $9,10^{\text {inv }}$ & $\mathrm{AF}$ & $\mathrm{A}, \mathrm{V}$ & & \\
\hline Todirostrum cinereum & & $9,10^{\text {inv }}$ & & $\mathrm{A}, \mathrm{V}$ & & \\
\hline Poecilotriccus plumbeiceps & 1 & $7,9,10$ & & A & & Forest \\
\hline Myiornis auricularis & 1 & 10 & $\mathrm{AF}$ & $\mathrm{P}$ & & Forest \\
\hline Hemitriccus diops & 1 & & $\mathrm{AF}$ & & & Forest \\
\hline Hemitriccus orbitatus & 1 & 10 & $\mathrm{AF}$ & A & & Forest \\
\hline Hemitriccus nidipendulus & 1 & & $\mathrm{AF}$ & & & Forest \\
\hline \multicolumn{7}{|l|}{ Tyrannidae } \\
\hline Hirundinea ferruginea & 1 & $8,9,10$ & & $\mathrm{P}$ & & \\
\hline Euscarthmus meloryphus & 1 & & & & & \\
\hline Tyranniscus burmeisteri & 1 & & & & & Forest \\
\hline Camptostoma obsoletum & 1 & 9,10 & & $\mathrm{~A}, \mathrm{~V}$ & & \\
\hline Elaenia flavogaster & 1 & 8,10 & & $\mathrm{~A}, \mathrm{~V}$ & & \\
\hline Elaenia spectabilis & & $10^{\text {inv }}$ & & $\mathrm{P}$ & & \\
\hline Elaenia parvirostris & & $10^{\text {inv }}$ & & $\mathrm{P}$ & & \\
\hline Elaenia mesoleuca & 1 & 10 & & $\mathrm{~A}, \mathrm{~V}$ & & \\
\hline Elaenia chiriquensis & 1 & & & & & \\
\hline Elaenia obscura & 1 & 10 & & $\mathrm{~A}, \mathrm{~V}$ & & \\
\hline Myiopagis caniceps & 1 & & & & & Forest \\
\hline Myiopagis viridicata & 1 & 9,10 & & $\mathrm{~A}, \mathrm{~V}$ & & Forest \\
\hline Capsiempis flaveola & 1 & 10 & & $\mathrm{P}$ & & Forest \\
\hline Phaeomyias murina & 1 & & & & & \\
\hline Phyllomyias virescens & 1 & 10 & $\mathrm{AF}$ & $\mathrm{R}$ & & Forest \\
\hline Phyllomyias fasciatus & 1 & 10 & & $\mathrm{~A}, \mathrm{~V}$ & & Forest \\
\hline Culicivora caudacuta & 1 & & & & & \\
\hline Serpophaga nigricans & 1 & & & & & \\
\hline Serpophaga subcristata & 1 & & & $\mathrm{~A}, \mathrm{~V}$ & & \\
\hline Attila rufus & 1 & 7,10 & $\mathrm{AF}$ & $\mathrm{A}, \mathrm{V}$ & & Forest \\
\hline Legatus leucophaius & 1 & & & & & \\
\hline Myiarchus swainsoni & 1 & $7,9,10$ & & $\mathrm{P}$ & & Forest \\
\hline Myiarchus ferox & & $9,10^{\text {inv }}$ & & $\mathrm{P}$ & & \\
\hline Myiarchus tyrannulus & 1 & 10 & & $\mathrm{~A}, \mathrm{~V}$ & & \\
\hline Sirystes sibilator & 1 & 10 & & A & & Forest \\
\hline Pitangus sulphuratus & 1 & $8,9,10$ & & $\mathrm{~A}, \mathrm{~V}$ & & \\
\hline Machetornis rixosa & & $8,9,10^{\text {inv }}$ & & $\mathrm{A}, \mathrm{V}$ & & \\
\hline Myiodynastes maculatus & 1 & $7,9,10$ & & $\mathrm{~A}, \mathrm{MN}, \mathrm{V}$ & & \\
\hline Megarynchus pitangua & 1,2 & 9,10 & & $\mathrm{~A}, \mathrm{~V}$ & & \\
\hline Myiozetetes similis & 1 & 9,10 & & $\mathrm{~A}, \mathrm{~V}$ & & \\
\hline Tyrannus melancholicus & 1,5 & $8,9,10$ & & $\mathrm{~A}, \mathrm{~V}$ & & \\
\hline Tyrannus savana & 1 & $8,9,10$ & & $\mathrm{P}$ & & \\
\hline Empidonomus varius & 1 & 9,10 & & $\mathrm{P}$ & & \\
\hline Conopias trivirgatus & 1 & & & & & Forest \\
\hline
\end{tabular}


Table 3. Continued...

\begin{tabular}{|c|c|c|c|c|c|c|}
\hline \multirow{2}{*}{ Taxon } & \multicolumn{2}{|c|}{ Record } & \multirow{2}{*}{ Endemism } & \multirow{2}{*}{ Evidence } & \multirow{2}{*}{ Threat status } & \multirow{2}{*}{ Habitat } \\
\hline & Historic & Recent & & & & \\
\hline Colonia colonus & 1,5 & 9,10 & & $\mathrm{~A}, \mathrm{~V}$ & & \\
\hline Myiophobus fasciatus & 1 & 9,10 & & $\mathrm{~A}, \mathrm{~V}$ & & \\
\hline Pyrocephalus rubinus & 1 & 9 & & & & \\
\hline Fluvicola albiventer & & $9^{\text {inv }}$ & & $\mathrm{A}, \mathrm{V}$ & & \\
\hline Fluvicola nengeta & & $9,10^{\text {inv }}$ & & $\mathrm{A}, \mathrm{V}$ & & \\
\hline Arundinicola leucocephala & & $8,9^{\text {inv }}$ & & $\mathrm{V}$ & & \\
\hline Gubernetes yetapa & & $8,9,10^{\text {inv }}$ & & $\mathrm{V}$ & & \\
\hline Alectrurus tricolor & 1 & & & & $\mathrm{VU}^{\mathrm{BR}}$ & \\
\hline Cnemotriccus fuscatus & 1 & & & & & Forest \\
\hline Lathrotriccus euleri & 1 & $7,9,10$ & & $P$ & & Forest \\
\hline Contopus cinereus & 1 & 10 & & $\mathrm{~A}, \mathrm{~V}$ & & \\
\hline Knipolegus cyanirostris & 1 & 10 & & $\mathrm{~V}$ & & \\
\hline Knipolegus lophotes & & $9,10^{\text {inv }}$ & & V & & \\
\hline Satrapa icterophrys & 1 & & & & & \\
\hline Xolmis cinereus & 1 & 9,10 & & V & & \\
\hline Xolmis velatus & & $8,10^{\text {inv }}$ & & $\mathrm{V}$ & & \\
\hline Muscipipra vetula & 1,3 & 10 & $\mathrm{AF}$ & $\mathrm{V}$ & & \\
\hline \multicolumn{7}{|l|}{ Vireonidae } \\
\hline Cyclarhis gujanensis & 1 & 9,10 & & $\mathrm{~A}, \mathrm{~V}$ & & \\
\hline Hylophilus amaurocephalus & & $10^{\text {inv }}$ & & $\mathrm{A}, \mathrm{V}$ & & \\
\hline Hylophilus poicilotis & 1 & 10 & $\mathrm{AF}$ & $\mathrm{A}, \mathrm{V}$ & & Forest \\
\hline Vireo olivaceus & 1 & $7,9,10$ & & $\mathrm{~A}, \mathrm{~V}$ & & \\
\hline \multicolumn{7}{|l|}{ Corvidae } \\
\hline Cyanocorax cristatellus & 1 & 10 & $\mathrm{CE}$ & $\mathrm{A}, \mathrm{V}$ & & \\
\hline \multicolumn{7}{|l|}{ Hirundinidae } \\
\hline Pygochelidon cyanoleuca & 1 & $8,9,10$ & & $\mathrm{~A}, \mathrm{~V}$ & & \\
\hline Alopochelidon fucata & 1 & & & & & \\
\hline Stelgidopteryx ruficollis & 1 & $8,9,10$ & & $\mathrm{~A}, \mathrm{~V}$ & & \\
\hline Progne tapera & & $9,10^{\text {inv }}$ & & $\mathrm{V}$ & & \\
\hline Progne chalybea & 1 & $8,9,10$ & & $\mathrm{~A}, \mathrm{~V}$ & & \\
\hline Tachycineta albiventer & 1 & & & & & \\
\hline Tachycineta leucorrhoa & 1 & 9 & & & & \\
\hline \multicolumn{7}{|l|}{ Troglodytidae } \\
\hline Troglodytes musculus & 1 & 9,10 & & $\mathrm{~A}, \mathrm{~V}$ & & \\
\hline Cistothorus platensis & 1 & & & & & \\
\hline \multicolumn{7}{|l|}{ Donacobiidae } \\
\hline Donacobius atricapilla & & $8,9,10^{\text {inv }}$ & & $\mathrm{P}$ & & \\
\hline \multicolumn{7}{|l|}{ Turdidae } \\
\hline Turdus leucomelas & 1 & 9,10 & & $\mathrm{~A}, \mathrm{MN}, \mathrm{V}$ & & \\
\hline Turdus rufiventris & 1 & 9,10 & & $\mathrm{~A}, \mathrm{MN}, \mathrm{V}$ & & \\
\hline Turdus amaurochalinus & 1 & 9,10 & & $\mathrm{~A}, \mathrm{~V}$ & & \\
\hline Turdus subalaris & & $10^{\text {inv }}$ & & A & & \\
\hline Turdus albicollis & 1 & $7,9,10$ & & $\mathrm{~A}, \mathrm{MN}, \mathrm{V}$ & & Forest \\
\hline \multicolumn{7}{|l|}{ Mimidae } \\
\hline Mimus saturninus & 1 & $8,9,10$ & & $\mathrm{~A}, \mathrm{~V}$ & & \\
\hline \multicolumn{7}{|l|}{ Motacillidae } \\
\hline Anthus lutescens & & $9^{\text {inv }}$ & & $\mathrm{A}, \mathrm{V}$ & & \\
\hline \multicolumn{7}{|l|}{ Passerellidae } \\
\hline Zonotrichia capensis & 1 & $8,9,10$ & & $\mathrm{~A}, \mathrm{~V}$ & & \\
\hline Ammodramus humeralis & 1 & $8,9,10$ & & $\mathrm{~A}, \mathrm{~V}$ & & \\
\hline Arremon semitorquatus & 1 & 7,10 & $\mathrm{AF}$ & $\mathrm{A}, \mathrm{V}$ & & Forest \\
\hline \multicolumn{7}{|l|}{ Parulidae } \\
\hline Setophaga pitiayumi & 1 & $7,9,10$ & & $\mathrm{~A}, \mathrm{~V}$ & & Forest \\
\hline Geothlypis aequinoctialis & 1,5 & $8,9,10$ & & $\mathrm{~A}, \mathrm{~V}$ & & \\
\hline Basileuterus culicivorus & 1 & $7,9,10$ & & $\mathrm{~A}, \mathrm{MN}, \mathrm{V}$ & & Forest \\
\hline Myiothlypis flaveola & & $9,10^{\text {inv }}$ & & $\mathrm{MN}, \mathrm{P}$ & & \\
\hline Myiothlypis leucoblephara & 1 & $7,9,10$ & & $\mathrm{~A}, \mathrm{MN}, \mathrm{V}$ & & Forest \\
\hline Myiothlypis rivularis & 1 & & & & & \\
\hline
\end{tabular}


Table 3. Continued...

\begin{tabular}{|c|c|c|c|c|c|c|}
\hline \multirow{2}{*}{ Taxon } & \multicolumn{2}{|c|}{ Record } & \multirow{2}{*}{ Endemism } & \multirow{2}{*}{ Evidence } & \multirow{2}{*}{ Threat status } & \multirow{2}{*}{ Habitat } \\
\hline & Historic & Recent & & & & \\
\hline \multicolumn{7}{|l|}{ Icteridae } \\
\hline Psarocolius decumanus & 1 & 10 & & $\mathrm{~A}, \mathrm{~V}$ & & Forest \\
\hline Icterus pyrrhopterus & & $10^{\text {inv }}$ & & $\mathrm{A}, \mathrm{V}$ & & \\
\hline Gnorimopsar chopi & 1 & & & & & \\
\hline Agelasticus cyanopus & & $8,10^{\text {inv }}$ & & $\mathrm{P}$ & & \\
\hline Chrysomus ruficapillus & & $9,10^{\text {inv }}$ & & $\mathrm{P}$ & & \\
\hline Pseudoleistes guirahuro & & $9,10^{\text {inv }}$ & & $\mathrm{A}, \mathrm{V}$ & & \\
\hline Molothrus oryzivorus & 1 & & & & & \\
\hline Molothrus bonariensis & 1 & 9,10 & & $\mathrm{~A}, \mathrm{~V}$ & & \\
\hline Sturnella superciliaris & & $8,9^{\text {inv }}$ & & $\mathrm{A}, \mathrm{V}$ & & \\
\hline \multicolumn{7}{|l|}{ Thraupidae } \\
\hline Pipraeidea melanonota & 1 & 10 & & $\mathrm{~V}$ & & Forest \\
\hline Cissopis leverianus & 1 & & & & & Forest \\
\hline Schistochlamys ruficapillus & 1 & & & & & \\
\hline Tangara seledon & 1 & & $\mathrm{AF}$ & & & Forest \\
\hline Tangara cyanoventris & 1 & & $\mathrm{AF}$ & & & Forest \\
\hline Tangara sayaca & 1 & $8,9,10$ & & $\mathrm{~A}, \mathrm{~V}$ & & \\
\hline Tangara palmarum & & $9,10^{\text {inv }}$ & & $\mathrm{A}, \mathrm{V}$ & & \\
\hline Tangara ornata & 1 & & $\mathrm{AF}$ & & & \\
\hline Tangara cayana & 1 & 9,10 & & $\mathrm{~A}, \mathrm{~V}$ & & \\
\hline Nemosia pileata & & $10^{\text {inv }}$ & & $\mathrm{A}, \mathrm{V}$ & & \\
\hline Conirostrum speciosum & & $9,10^{\text {inv }}$ & & $\mathrm{R}$ & & \\
\hline Sicalis flaveola & 1 & 10 & & $\mathrm{~A}, \mathrm{~V}$ & & \\
\hline Haplospiza unicolor & 1 & & $\mathrm{AF}$ & & & Forest \\
\hline Hemithraupis ruficapilla & 1 & 7,10 & $\mathrm{AF}$ & $\mathrm{P}$ & & Forest \\
\hline Volatinia jacarina & 1 & $8,9,10$ & & $\mathrm{~A}, \mathrm{~V}$ & & \\
\hline Trichothraupis melanops & 1 & $7,9,10$ & & $\mathrm{~A}, \mathrm{MN}, \mathrm{V}$ & & Forest \\
\hline Coryphospingus cucullatus & 1 & 9,10 & & $\mathrm{~A}, \mathrm{~V}$ & & \\
\hline Tachyphonus coronatus & 1 & $7,9,10$ & & $\mathrm{MN}, \mathrm{P}$ & & Forest \\
\hline Ramphocelus carbo & & $10^{\text {inv }}$ & & $\mathrm{P}$ & & \\
\hline Tersina viridis & 1 & 10 & & $\mathrm{~A}, \mathrm{~V}$ & & \\
\hline Dacnis nigripes & 1 & & $\mathrm{AF}$ & & & Forest \\
\hline Dacnis cayana & 1 & 9,10 & & $\mathrm{~A}, \mathrm{~V}$ & & \\
\hline Coereba flaveola & 1 & 9,10 & & $\mathrm{~A}, \mathrm{~V}$ & & \\
\hline Sporophila lineola & & $9,10^{\text {inv }}$ & & $\mathrm{A}, \mathrm{V}$ & & \\
\hline Sporophila caerulescens & 1,5 & $8,9,10$ & & $\mathrm{P}$ & & \\
\hline Sporophila leucoptera & & $10^{\text {inv }}$ & & $\mathrm{A}, \mathrm{V}$ & & \\
\hline Sporophila angolensis & 1 & 10 & & $\mathrm{~A}, \mathrm{~V}$ & & \\
\hline Coryphaspiza melanotis & 1 & & $\mathrm{CE}$ & & $\mathrm{EN}^{\mathrm{BR}}$ & \\
\hline Emberizoides herbicola & 1 & 10 & & $\mathrm{~A}, \mathrm{~V}$ & & \\
\hline Saltator similis & 1 & $7,8,9,10$ & & $\mathrm{~A}, \mathrm{~V}$ & & Forest \\
\hline Saltator fuliginosus & 1 & 10 & $\mathrm{AF}$ & $\mathrm{A}, \mathrm{V}$ & & Forest \\
\hline Thlypopsis sordida & & $9,10^{\text {inv }}$ & & $\mathrm{A}, \mathrm{MN}, \mathrm{V}$ & & \\
\hline Pyrrhocoma ruficeps & 1 & $7,9,10$ & & $\mathrm{~V}$ & & Forest \\
\hline \multicolumn{7}{|l|}{ Cardinalidae } \\
\hline Piranga flava & 1 & 9 & & & & \\
\hline Habia rubica & 1 & $7,9,10$ & & $\mathrm{~A}, \mathrm{~V}$ & & Forest \\
\hline Cyanoloxia glaucocaerulea & & $10^{\text {inv }}$ & & $\mathrm{P}$ & & \\
\hline Cyanoloxia brissonii & 1 & 9,10 & & $\mathrm{~V}$ & & \\
\hline \multicolumn{7}{|l|}{ Fringillidae } \\
\hline Spinus magellanicus & 1 & 9,10 & & $\mathrm{~V}$ & & \\
\hline Euphonia chlorotica & 1 & 9,10 & & $\mathrm{~A}, \mathrm{~V}$ & & \\
\hline Euphonia violacea & 1 & & & & & \\
\hline Euphonia chalybea & 1 & & $\mathrm{AF}$ & & & \\
\hline Euphonia cyanocephala & 1 & 10 & & $\mathrm{~V}$ & & Forest \\
\hline Euphonia pectoralis & 1 & & $\mathrm{AF}$ & & & \\
\hline Chlorophonia cyanea & 1 & & & & & \\
\hline
\end{tabular}


Table 3. Continued.

\begin{tabular}{|c|c|c|c|c|c|c|}
\hline \multirow{2}{*}{ Taxon } & \multicolumn{2}{|c|}{ Record } & \multirow{2}{*}{ Endemism } & \multirow{2}{*}{ Evidence } & \multirow{2}{*}{ Threat status } & \multirow{2}{*}{ Habitat } \\
\hline & Historic & Recent & & & & \\
\hline \multicolumn{7}{|l|}{ Estrildidae } \\
\hline Estrilda astrild & & $9^{\text {inv }}$ & & $\mathrm{A}, \mathrm{V}$ & & \\
\hline \multicolumn{7}{|l|}{ Passeridae } \\
\hline Passer domesticus & & $8,9,10^{\text {int }}$ & & $\mathrm{A}, \mathrm{V}$ & & \\
\hline
\end{tabular}

Table 4. Jaccard Dissimilarity Indices comparing bird species richness between eight protected forests in São Paulo, southern Brazil. INF 1 refers to historical species richness, whereas INF 2 refers to recent species richness. Ipanema National Forest (INF), Intervales State Park (ISP), Carlos Botelho State Park (CBSP), Caetetus Ecological Station (CES), Lençóis Paulista (LP), Serra do Mar State Park (SMSP), Boraceia Biological Station (BBS), Barreiro Rico Farm (BRF).

\begin{tabular}{cccccccc}
\hline & ISP & CBSP & INF 1 & INF 2 & CES & LP & SMSP \\
\hline CBSP & 0.400 & & & & & \\
INF 1 & 0.453 & 0.587 & & & & \\
INF 2 & 0.583 & 0.633 & 0.559 & & & \\
CES & 0.551 & 0.615 & 0.526 & 0.417 & & \\
LP & 0.639 & 0.689 & 0.578 & 0.399 & 0.363 & 0.653 & \\
SMSP & 0.397 & 0.548 & 0.511 & 0.603 & 0.572 & 0.647 & 0.462 \\
BBS & 0.320 & 0.416 & 0.470 & 0.581 & 0.571 & 0.511 & \\
BRF & 0.595 & 0.626 & 0.626 & 0.511 & 0.458 & 0.622 \\
\hline
\end{tabular}

such as seed dispersal (Galetti et al. 2013), maintenance of the original vegetation structure may be seriously compromised at INF in the long term.

Pre- and post-isolation comparisons of tropical forests show the percentage of extinct species varies according to the time lag between isolation. It varies from $49 \%$ in a 4-ha fragment in Singapore over 100 years (Sodhi et al. 2005 ) to ca $30 \%$ after 50-90 years in Colombian forests (Kattan et al. 1994, Renfijo 1999) and Barro Colorado Island, Panama (Robinson 1999). For the Atlantic Forest, Aleixo \& Vielliard (1995) noted that $54 \%$ of the forest avifauna of an isolated 251 ha southeastern Brazilian remnant was extinct after a 15-year interval. Authors who compared historical and recent data show a slightly better scenario. Christiansen \& Pitter (1997) showed that almost $17 \%$ of all forest species were extinct from fragments in Lagoa Santa, southeastern Brazil (original data compiled from Krabbe [2007]), after 130 years. Willis \& Oniki (2002), Ribon et al. (2003) and Silveira (2009) reported similar percentages (20.4-30\%) of forest bird extinctions for Santa Teresa and Santo André, southeastern Brazil, after 50, 70 and 106 years separating deforestation time-lags, respectively.

\subsection{Climate change}

It is expected that habitat loss will act synergistically with climate change in the next decades, being major threats to biodiversity and human wellbeing (Pecl et al. 2017). Climate change has been suggested to reduce survival rates of Neotropical bird species (Blake \& Loiselle 2015) and to reduce species richness and their current ranges both in the Atlantic Forest (Anciães \& Peterson 2006) and Cerrado (Marini et al. 2009), as well as in several other regions in the planet (Şekercioğlu et al. 2012). Although the effects of climate change on species richness composition between periods must be considered, due to lack of detailed surveying methods during the 1800 s, we cannot account for climatic changes on bird communities.

\section{Changes in specific species}

\subsection{Extinctions}

Although local extinction is quite probable, one cannot simply assume it (Scheffers et al. 2011) because three forest species collected by Natterer had no record until the second last survey we conducted at the INF in 2012. This is the case of a single recording of the Shrike-like Cotinga
Laniisoma elegans (Thunberg 1823), and VC's aural and visual record of the Olivaceous Elaenia Elaenia mesoleuca (Deppe 1830). During our last visit, LFAF heard the Squamate Antbird Myrmoderus squamosus song, after a 190-year span. Robinson (1999) also reported similar re-encounters, although for a shorter ( 25 years) time span.

It is also important to highlight one of the most important records at INF, the Eskimo Curlew Numenius borealis (Forster 1772). Once abundant, it has not been recorded with certainty since 1963, and none have been confirmed on wintering grounds since 1939 (BirdLife International 2015). The species exhibited an elliptical migratory route connecting its Arctic breeding grounds in North America to its wintering range in southern South America. Besides the specimen collected by Natterer at the INF, there are other specimens from the states of Mato Grosso and Amazonas, the only indications of its presence in Brazilian grounds (Straube 2008).

\subsection{Recent records due to habitat change}

It is intriguing that Natterer did not collect Dysithamnus mentalis (Temminck 1823) or Todirostrum poliocephalum (Wied 1831), both currently abundant at the INF. These forest edge species are quite common in early to medium successional stages of vegetation and are found, though more scantly, even in mature forests of the state as well. We suspect they must have been very rare hundreds of years ago, when pristine forests predominated the surroundings. A similar phenomenon was observed at Serra da Cantareira, where Herpsilochmus rufimarginatus (Temminck 1822) used to be rare and is suggested to be one of the most abundant species in a time span of only two decades (Tonetti et al. 2017). Another noteworthy absence during the nineteenth century is Furnarius rufus (Gmelin 1788), which probably began invading eastern parts of the country after large deforestation in western Brazil took place during the 1800s (Sick 1997).

In the case of some marsh birds, we assume there were no southern cattail Typha domingencis (Pers.) or bulrush Scirpus californicus (C. A. Mey) Steud marshes while Natterer stayed at the INF. Several typical marsh species such as Laterallus melanophaius (Vieillot 1819), Phacellodomus ferrugineigula (Pelzeln 1858), Arundinicola leucocephala (Linnaeus 1764), Agelasticus cyanopus (Vieillot 1819) and Chrysomus ruficapillus (Vieillot 1819) are missing from this period. Natterer probably would have collected these conspicuous species. Some species can help illustrate what kind of aquatic environments were present at the time. We suspect these were riverside 
vegetation because of the presence of Anhima cornuta (Linnaeus 1766) and Serpophaga nigricans (Vieillot 1817), while wet or flooded grasslands must have been visited, as suggested by the several Herons, Ibises and migratory Sandpipers.

\section{Museum collections}

Within the climax of both digital and molecular century, we take great pleasure in highlighting the importance of traditional museum skin collections. A special issue on museum collections effects on bird diversity and extinction did detail reasons for continued growth of collections (Collar et al. 2003). In addition, natural history collections were evidenced as sources of data that can complement past surveys. They also comprise samples that span the period of accelerated anthropogenic habitat destruction and climate warming, reflecting baseline conditions (Lister 2011). There could not be a comparison to be made without Natterer's committed bird collections and Pelzeln's (1868) publication, rendering our study a rare opportunity to evaluate how bird species richness differed within the same site almost 200 years apart.

\section{Supplementary material}

The following online material is available for this article:

Appendix I - Historical survey

Appendix II - Recent literature

\section{Acknowledgments}

We wish to thank Holcim Brasil on behalf of L. C. B. Nepomuceno, who invited us to carry out this Fauna Monitoring Program. J. F. Pacheco shared with us crucial information about Langsdorff's voyages to Brazil. P. S. Tomkovich, curator of the Zoological Museum of the Moscow State University, generously detailed Langsdorff's Brazilian bird collections deposited under his care. A. Whittaker identified M. Cohn-Haft's recording of the Shrike-like Cotinga and both kindly agreed in sharing their remarkable record with us. G. Del-Rio, F. C. Straube, A. Zillikens, T. Putker and two anonymous reviewers contributed with important remarks. A. A. Kuniy (JGP Consultoria e Participações Ltda.) made this paper possible. VC thanks IdeaWild and LFS receives a grant from the Brazilian Council of Research (CNPq).

\section{Author Contributions}

Vagner Cavarzere: Substantial contribution in the concept and design of the study, contribution to data collection, contribution to data analysis and interpretation, contribution to manuscript preparation, contribution to critical revision, adding intellectual content.

Luís Fábio Silveira: Substantial contribution in the concept and design of the study, contribution to data analysis and interpretation, contribution to manuscript preparation, contribution to critical revision, adding intellectual content.

Vinicius Rodrigues Tonetti: Substantial contribution in the concept and design of the study, contribution to data analysis and interpretation, contribution to manuscript preparation, contribution to critical revision, adding intellectual content.

Pedro Ferreira Develey: Contribution to data analysis and interpretation, contribution to critical revision, adding intellectual content.

Flávio Kulaif Ubaid: Contribution to data collection, contribution to data analysis and interpretation, contribution to manuscript preparation, contribution to critical revision, adding intellectual content.

Luciano Bonatti Regalado: Contribution to data collection.

Luiz Fernando de Andrade Figueiredo: Contribution to data collection.

\section{Conflicts of interest}

The authors declare that they have no conflict of interest related to the publication of this manuscript.

\section{References}

ABRAMOV, A.V. \& BARANOVA, G.I. 2008. The Langsdorff's expedition to Brazil and its mammal collection kept in the Zoological Institute of St. Petersburg, Russia. Russ. J. Theriol. 7(1):41-50.

ALEIXO, A. \& VIELLIARD, J.M.E. 1995. Composição e dinâmica da avifauna da Mata de Santa Genebra, Campinas, São Paulo, Brasil. Rev. Bras. Zool. 12(3):493-511.

ANCIÃES, M. \& PETERSON, T. 2006. Climate change effects on Netropical Manakin diversity based on ecological niche modelling. Condor 108(4):778-791.

ANTUNES, A.Z., SILVA, B.G.D., MATSUKUNA, C.K., ESTON, M.R.D. \& SANTOS, A.M.R.D. 2013. Aves do Parque Estadual Carlos Botelho-SP. Biota Neotrop. 13(2):124-140. http://dx.doi.org/10.1590/S1676-06032013000200012 (last access on 07/07/2017)

BARNOSKY, A.D., MATZKE, N., TOMIYA, S., WOGAN, G.O., SWARTZ, B., QUENTAL, T.B., MARSHALL, C., MCGUIRE, J.L., LINDSEY, E.L., MAGUIRE, K.C., MERSEY, B. \& FERRER, E.A. 2011. Has the Earth's sixth mass extinction already arrived? Nature 471(7336): 51-57.

BERTHELS, D.E., KOMISSAROV, B.N. \& LYSENKO, T.I. 1979. Materialien der Brasilien-Expedition 1821-1829 des Akademiemitgliedes Georg Heinrich Freiherr von Langsdorff (Grigorij Ivanoviéc Langsdorff): vollständige wissenschaftliche Beschreibung. Dietrich Reimer, Berlin.

BIBBY, C.J., BURGESS, N.D., HILL, D.A. \& MUSTOE, S.H. 2000. Bird census techniques. Elsevier, Amsterdam.

BIERREGAARD, R.O. \& LOVEJOY, T.E. 1989. Effects of forest fragmentation on Amazonian understory bird communities. Acta Amaz. 19:215-241.

BirdLife International. 2015. Numenius borealis. The IUCN Red List of Threatened Species. Version 2016.3. http://www.iucnredlist.org (last access in 24/02/2017)

BLAKE, J.G. \& LOISELLE, B.A. 2015. Enigmatic declines in bird numbers in lowland forest of eastern Ecuador may be a consequence of climate change. PeerJ 3:e1177.

BLAKE, J.G. \& LOISELLE, B.A. 2016. Long-term changes in composition of bird communities at an "undisturbed" site in eastern Ecuador. Wilson J. Ornithol. 128(2): 255-267.

BRANDT, J.F. 1835. Mammalium Rodentium Exoticorum Novorum vel Minus Rite Cognitorum Musei Academici Zoologici, Descriptio et Icones. Mém. Acad. Imp. Sci. Saint-Pétersbourg 6(1):358-442.

BUTCHART, S.H., WALPOLE, M., COLLEN, B., VAN STRIEN, A., SCHARLEMANN, J.P., ALMOND, R.E., BAILLIE, J.E., BOMHARD, B., BROWN, C., BRUNO, J., CARPENTER, K.E., CARR, G.M., CHANSON, J., CHENERY, A.M., CSIRKE, J., DAVIDSON, N.C., DENTENER, F., FOSTER, M., GALLI, A., GALLOWAY, J.N., GENOVESI, P., GREGORY, R.D., HOCKINGS, M., KAPOS, V., LAMARQUE, J.F., LEVERINGTON, F., LOH, J., MCGEOCH, M.A., MCRAE, L., MINASYAN, A., MORCILLO, M.H., OLDFIELD, T.E., PAULY, D., QUADER, S., REVENGA, C., SAUER, J.R., SKOLNIK, B., SPEAR, D., STANWELL-SMITH, D., STUART, S.N., SYMES, A., TIERNEY, M., TYRRELL, T.D., VIÉ, J.C. \& WATSON, R. 2010. Global biodiversity: indicators of recent declines. Science. 328(5982): 1164-1168.

CAVARZERE, V., MORAES, G.P. \& DONATELLI, R.J. 2009. Avifauna da Estação Ecológica dos Caetetus, interior de São Paulo, Brasil. Pap. Avulsos Zool. 49(35):477-485.

CAVARZERE, V., MORAES, G.P. \& SILVEIRA, L.F. 2010. Boracéia Biological Station: an ornithological review. Pap. Avulsos Zool. 50(13):189-201.

CAVELIER, J., AIDE, T.M., SANTOS, C., EUSSE, A.M., DUPUY, J.M. 1998. The savannization of moist forests in the Sierra Nevada de Santa Marta, Colombia. J. Biogeogr. 25(5):901-912.

CEBAllos, G., EHRLICH, P.R., BARNOSKY, A.D., GARCIA, A., PRINGLE, R.M. \& PALMER, T.M. 2015. Accelerated modern human-induced species losses: Entering the sixth mass extinction. Science Advances 1:e1400253-e1400253. 
CEBALLOS, G., EHRLICH, P.R., DIRZO, R. 2017. Biological annihilation via the ongoing sixth mass extinction signaled by vertebrate population losses and declines. Proc. Natl. Acad. Sci. 114(30):1-8.

CHRISTIANSEN, M.B. \& PITTER, E. 1997, Species loss in a forest bird community near Lagoa Santa in southeastern Brazil. Biol. Conserv. 80(1):23-32.

COLLAR, N., FISHER, C., FEARE, C. 2003. Why museums matter: Avian archives in an age of extinction. Bull. Br. Orn. Club. 123A:360 p.

CURTIS, J.R. \& ROBINSON, W.D. 2015. Sixty years of change in avian communities of the Pacific Northwest. PeerJ 3:e1152.

DEL HOYO, J., ELLIOT, A. \& SARGATAL, J. 1992-2011. Handbook of the Birds of the World. Lynx Editions, Barcelona.

DIRZO, R., YOUNG, H.S., GALETTI, M., CEBALlO, G., ISAAC, N.J. \& COLLEN, B. 2014. Defaunation in the Anthropocene. Science 345(6195):401-406.

DONATELLI, R.J., COSTA, T.V.V. \& FERREIRA, C.D. 2004. Dinâmica da avifauna em fragmento de mata na Fazenda Rio Claro, Lençóis Paulista, São Paulo, Brasil. Rev. Bras. Zool. 21(1):97-114.

DUPRÉ, L. 1885. Memória sobre a Fábrica de Ferro de São João do Ypanema. Mariana (Brazil). Anais da Escola de Minas de Ouro Preto. Typografia D’O Germinal, Mariana.

GALETTI, M., GUEVARA, R., CÔRTES, M.C., FADINI, R., VON MATTER, S., LEITE, A.B., LABECCA, F., RIBEIRO, T., CARVALHO, C.S., COLLEVATTI, R.G., PIRES, M.M., GUIMARÃES jr, P.R., BRANCALION, P.H., RIBEIRO, M.C \& JORDANO, P. 2013. Functional extinction of birds drives rapid evolutionary changes in seed size. Science. 340(6136):1086-1090.

GRANZINOLLI, M.A.M. 2009. Ibycter americanus (Boddaert, 1783). In Fauna ameaçada de extinção no Estado de São Paulo: Vertebrados (P.M. Bressan, M.C.M. Kierulff \& A.M. Sugieda, eds). Fundação Parque Zoológico de São Paulo/SEMA, São Paulo, p. 144

HELLMAYR, C.E. 1906. Revision der Spix'schen typen brasilianischer vögel. Abh. Mat.-Phys. K., K. Bayer Akad. Wiss. 22:561-726.

JOHNSON, C.N., BALMFORD, A., BROOK, B.W., BUETTEL, J.C., GALETTI, M., GUANGCHUN, L. \& WILMSHURST, J.M. Biodiversity losses and conservation responses in the Anthropocene. Science 356(6335):270-275.

KATTAN, G.H., ALVAREZ-LOPES, H. \& GIRALDO, M. 1994. Forest fragmentation and bird extinctions: San Antonio eighty years later. Conserv, Biol. 8(1):138-146.

KRABBE, N. 2007. Birds collected by P. W. Lund and J. T. Reinhardt in southeastern Brazil between 1825 and 1855, with notes on P. w. Lund's travels in Rio de Janeiro. Rev. Bras. Ornitol. 15(3):331-357.

LECK, C.F. 1979. Avian extinctions in an isolated tropical wet forest preserve, Ecuador. Auk. 96(2):343-352.

LISTER, A.M. 2011. Natural history collections as sources of long-term datasets. Trends Ecol. Evol. 26(4): 153-154.

LOPES, L.E., VASCONCELOS, M.F., GONZAGA, L.P. 2017. A cryptic new species of hummingbird of the Campylopterus largipennis complex (Aves: Trochilidae). Zootaxa 4268(1):1-33.

MAGALHÃES, J.C.R. 1999. As aves na Fazenda Barreiro Rico. Plêiade, São Paulo.

MARINI, M.Â., BARBET-MASIN, M., LOPES, L.E., JIGUET, F. 2009. Predicted climate-driven bird distribution changes and forecasted conservation conflicts in a Neotropical savanna. Conserv. Biol. 23(6):1558-1567.

MENETRIES, E. 1835 Monographie de la Famille des Myiotherinae où Sont Décrites les Espèces qui Ornent le Musée de l'Académie Impériale des Sciences. Mém. Acad. Imp. Sci. Saint-Pétersbourg 6:443-543.

MENON, O.N. 1992 A real fábrica de ferro de São João do Ipanema e seu mundo-1811-1835. Dissertação de Mestrado, Pontifícia Universidade Católica de São Paulo, São Paulo.

MIKAN, J.C. 1820. Delectus florae et faunae Brasiliensis: jussu et auspiciis Francisi I, Austriae imperatoris investigatae. Sumptubus auctoris, Vindobonae.

MOURA, N.G., LEES, A.C., ALEIXO, A., BARLOW, J., DANTAS, S.M., FERREIRA, J., LIMA, M.F.C.\& GARDNER, T.A. 2014. Two hundred years of local avian extinctions in eastern Amazonia. Conserv. Biol. 28(5):1271-1281.

MMA-Ministério do Meio Ambiente. 2003. Plano de Manejo da Floresta Nacional de Ipanema. Ministério do Meio Ambiente, Sorocaba.
PELZELN, A. 1868. Zur Ornithologie Brasiliens. Resultate von Johann Natterers Reisen in den Jahren 1817 bis 1835. Druck Verlag A Pichler's Witwe \& Sohn, Wien.

PIACENTINI, V.Q., ALEIXO, A., AGNE, C.E., MAURÍCIO, G.N., PACHECO, J.F., BRAVO, G.A., BRITO, G.R.R., NAKA, L.N., OLMOS, F., POSSO, S., SILVEIRA, L.F., BETINI, G.S., CARRANO, E., FRANZ, I., LEES, A., LIMA, L.M., PIOLI, D., SCHUNCK, F., AMARAL, F.R., BENCKE, G.A., COHN-HAFT, M., FIGUEIREDO, L.F., STRAUBE, F.C. \& CESARI, E. 2015. Annotated checklist of the birds of Brazil by the Brazilian Ornithological Records Committee / Lista comentada das aves do Brasil pelo Comitê Brasileiro de Registros Ornitológicos. Rev. Bras. Ornitol. 23(2):91-298.

PARKER, T.A, STOTZ, D.F.\& FITZPATRICK, J.W. 1996. Ecological and distributional databases. In: Neotropical birds: ecology and conservation (D.F. Stotz, J.W. Fitzpatrick, T.A. Parker \& D.K. Moskovits, eds). The University of Chicago Press, Chicago, p. 118-436.

PECL, G.T., ARAÚJO, M.B., BELL, J.D., BLANCHARD, J., BONEBRAKE, T.C., CHEN, I-C., CLARK, T.D., COLWELL, R.K., DANIELSEN, F., EVENGÅRD, B., FALCONI, L., FERRIER, S., FRUSHER, S., GARCIA, R.A., GRIFFIS, R.B., HOBDAY, A.J., ANION-SCHEEPERS, C., JARZYNA, M.A., JENNINGS, S., LENOIR, J., LINNETVED, H.I., MARTIN, V.Y., MCCORMACK, P.C., MCDONALD, J., MITCHELL, N.J., MUSTONEN, T., PANDOLFI, J.M., PETTORELLI, N., POPOVA, E., ROBINSON, S.A., SCHEFFERS, B.R., SHAW, J.D., SHAW, J.D., SORTE, C.J.B., STRUGNELL, J.M., SUNDAY, J.M., TUANMU, M.N., VERGÉS, A., VILLANUEVA, C., WERNBERG, T., WAPSTRA, E., WILLIAMS, S.E. 2017. Biodiversity redistribution under climate change: Impacts on ecosystems and human wellbeing. Science 355(6332):eaai9214

PIMM, S.L., JENKINS, C.N., ABELL, R., BROOKS, T.M., GITTLEMAN, J.L., JOPPA, L.N., RAVEN, P.H., ROBERTS, C.M. \& SEXTON, J.O. 2014. The biodiversity of species and their rates of extinction, distribution, and protection. Science 344(6187):1246752-1246752.

PINTO, O.M.O. 1979. A ornitologia do Brasil através das idades (século XVI a século XIX). Revista dos Tribunais S/A, São Paulo.

R Development Core Team (2015) R: A language and environment for statistical computing. R Foundation for Statistical Computing. Vienna, Austria. http:// www.R-project.org.

RADAMBRASIL. 1983. Levantamento dos recursos naturais. Ministério de Minas e Energia, Rio de Janeiro.

REGALADO, L.B. 1999. Composição e distribuição de aves passeriformes em uma parcela de mata do Morro de Araçoiaba (Floresta Nacional de Ipanema, Iperó/SP) utilizando um sistema de informação geográfica. Dissertação de Mestrado, Escola de Engenharia de São Carlos, São Carlos.

REGO, M.A., MOREIRA-LIMA, L., SILVEIRA, L.F. \& FRAHNERT, S. 2013. On the ornithological collection of Friedrich Sellow in Brazil (1814-1831), with some considerations about the provenance of his specimens. Zootaxa. 3616(5):478-484.

REINHARDT, J. 1870. Bidrag til Kundskab om Fuglefaunaen i Brasilien campos. Videnskabelige Meddelelser fra den naturhistoriske Forening I Köbenhavn $1-7: 1-124$.

RENFIJO, L.M. 1999. Composition Changes in a Subandean Avifauna after LongTerm Forest Fragmentation. Conserv. Biol. 13(5):1124-1139.

RIBEIRO, M.C., METZGER, J.P., MARTENSEN, A.C., PONZONI, F.J. \& HIROTA, M.M. 2009. The Brazilian Atlantic Forest: how much is left and how is the remaining forest distributed? Implications for conservation. Biol. Conserv. 142(6):1141-1153.

RIBON, R., SIMON, J.E. \& MATTOS, G.T. 2003. Bird extinctions in Atlantic Forest fragments of the Viçosa region, southeastern Brazil. Conserv. Biol. 17(6):1827-1839.

ROBINSON, W.D. 1999. Long-term changes in the Avifauna of Barro Colorado Island, Panama, a tropical forest isolate. Conserv. Biol. 13(1):85-97.

SAINT-HILAIRE A. 1976. Viagem à Província de São Paulo. EDUSP, São Paulo. SCHEFFERS, B.R., YONG, .D.L., HARRIS, J.B.C, GIAM, X. \& SODHI, N.S. 2011 .The world's rediscovered species: back from the brink? PlosOne 6(7):e22531.

ŞEKERCIOĞLU, C.H., PRIMACK, R.B. \& WORMWORTH, J. 2012. The effects of climate change on tropical birds. Biol. Conserv. 148(1):1-18. 
SICK, H. 1997. Ornitologia Brasileira. Nova Fronteira Rio de Janeiro.

SILVA, C. 2010. Alterações na comunidade de aves do Morro de Araçoiaba (Floresta Nacional de Ipanema - FLONA, Iperó, SP). Rev. Conhecimento Online 2(2):1-36.

SILVA, J.M.C. \& BATES, J.M. 2002. Biogeographic patterns and conservation in the South American Cerrado: a tropical savanna hotspot. BioScience 52(3):225-233.

SILVA, C. \& REGALADO, L.B. 1998. Nidificação em cavidade rochosa por uruburei, Sarcoramphus papa (Falconiformes: Cathartidae) no Morro de Araçoiaba (Floresta Nacional de Ipanema - Iperó, São Paulo). Boletim CEO 13:4-8.

SILVEIRA, L.F. 2009. As aves: uma revisão histórica do conhecimento ornitológico em uma Reserva de Mata Atlântica do Estado de São Paulo. In Patrimônio da Reserva Biológica do Alto da Serra de Paranapiacaba. A antiga Estação Biológica do Alto da Serra (M.I.M.S. Lopes, M. Kirizawa \& M.M.R.F. Melo, eds). Instituto de Botânica, São Paulo, p. 623-636.

SILVEIRA, L.F.\& D'HORTA, F.M. 2001. Avifauna da região de Vila Bela da Santíssima Trindade, Mato Grosso. Pap. Avulsos Zool. 42(10):265-286.

SILVEIRA, L.F. \& UEZU. A. 2011. Checklist das aves do Estado de São Paulo, Brasil. Biota Neotrop. 11(1a):83-110. http://dx.doi.org/10.1590/S167606032011000500006 (last access on 07/07/2017)

SIMPSON, R., CAVARZERE, V. \& SIMPSON, E. 2012. List of documented bird species from the municipality of Ubatuba, state of São Paulo, Brazil. Pap. Avulsos Zool. 52(21):233-254.

SODHI, N.S., LEE, T.M., KOH, L.P. \& DUNN, R.R. 2005. A century of avifaunal turnover in a small tropical rainforest fragment. Anim. Conserv. 8(2):217--222.

SPIX, J.B. \& MARTIUS, C.F.P. 1840. Avium species novae, quas in itinere per Brasliliam annis MDCCCXVII-MDCCCXX jussu et auspiciis Maximilani Josephi I. Bavariae regis suscepto / collegit et descripsit Dr. J. B. de Spix. Impensis, Monachii.

SPIX, J.B. \& MARTIUS, C.F.P. 1824. Travels in Brazil, in the years 1817-1820. Undertaken by command of his majesty the king of Bavaria. Longman, Hurst, Rees, Orme, Brown, and Green, Paternoster-Row, London.

STOPIGLIA, R., STRAKER, L.C. \& RAPOSO, M.A. 2009. Kinglet Calyptura Calyptura cristata (Vieillot, 1818): documented record for the state of São Paulo and taxonomic status of the name Pipra tyrannulus Wagler, 1830. Bull. Br. Ornithol. Club 129(3):185-188.

STRASSBURG, B.B.N., BROOKS, T., FELTRAN-BARBIERI, R., IRIBARREM, A., CROUZEIlles, R., LOYOLA, R., LATAWIEC, A.E., OLIVEIRA
FILHO, F.J.B., SCARAMUZZA, C.A.M., SCARANA, F.R., SOARESFILHO, B. \& BALMFORD, A. 2017. Moment of truth for the Cerrado hotspot. Nat. Ecol. Evol. 1:0099.

STRAUBE, F.C. 2008. Numenius borealis (Foster, 1772). In Livro vermelho da fauna brasileira ameaçada de extinção (A.B.M. Machado, G.M. Drummond \& A.P. Paglia, eds). MMA, Brasília, p. 450-451.

STRESEMANN, E. 1948. Der Naturfoscher Friedrich Sellow (1831) und sein Beitrag zur Kenntnis Brasiliens. Zool. Jahrb. Abt. Anat. Ontog. Tiere 77(6):401-425.

TONETTI, V.R. \& PIZO, M.A. 2016. Density and microhabitat preference of the Southern Bristle-Tyrant (Phylloscartes eximius): Conservation policy implications. Condor 118(4):791-803.

TONETTI, V.R., REGO, M.A., DE LUCA, A.C., DEVELEY, P.F., SCHUNCK, F. \& SILVEIRA, L.F. 2017. Historical knowledge, richness and relative representativeness of the avifauna of the largest native urban rainforest in the world. Zoologia 34: e13728.

VIELLIARD, J.M.E. \& SILVA, W.R. 2001. Avifauna. In Intervales: fundação para a conservação e a produção florestal do Estado de São Paulo (C. Leonel, ed). Fundação, São Paulo, p. 124-144.

WARD jr, J.H. 1963. Hierarchical grouping to optimize an objective function. J. Am. Stat. Assoc. 58(301):236-244.

WHELAN, C.J., WENNY, D.G. \& MARQUIS, R.J. 2008. Ecosystem services provided by birds. AAnn. N. Y. Acad. Sci. 1134(1): 25-60.

WILLIS, E.O. 1974. Populations and local extinctions of birds on Barro Colorado Island, Panama. Ecol. Monogr. 44(2):153-169.

WILLIS, E.O. 1979. The composition of avian communities in remanescent woodlots in southern Brazil. Pap. Avulsos Zool. 33(1):1-25.

WILLIS, E.O. \& ONIKI, Y. 2002. Birds of Santa Teresa, Espírito Santo, Brazil: do humans add or subtract species? Pap. Avulsos Zool. 42(9):193-264.

WILLIS, E.O. \& ONIKI, Y. 2003. Aves do Estado de São Paulo. Divisa, Rio Claro.

Received: 10/07/2017

Revised: 13/09/2017

Accepted: 10/10/2017

Published online: 30/10/2017 